\title{
A Comparison of Variant Theories of Intact Biochemical Systems. II. Flux-Oriented and Metabolic Control Theories*
}

\author{
ALBERT SORRIBAS ${ }^{\dagger}$ AND MICHAEL A. SAVAGEAU \\ Department of Microbiology and Immunology, The University of Michigan, Ann Arbor, \\ Michigan 48109-0620
}

Received 6 July 1988; revised 1 October 1988

\begin{abstract}
In the past two decades, several theories, all ultimately based upon the same power-law formalism, have been proposed to relate the behavior of intact biochemical systems to the properties of their underlying determinants. Confusion concerning the relatedness of these alternatives has become acute because the implications of these theories have never been compared. In the preceding paper we characterized a specific system involving enzymeenzyme interactions for reference in comparing alternative theories. We also analyzed the reference system by using an explicit variant that involves the S-system representation within biochemical systems theory (BST). We now analyze the same reference system according to two other variants within BST. First, we carry out the analysis by using an explicit variant that involves the generalized mass action representation, which includes the flux-oriented theory of Crabtree and Newsholme as a special case. Second, we carry out the analysis by using an implicit variant that involves the generalized mass action representation, which includes the metabolic control theory of Kacser and his colleagues as a special case. The explicit variants are found to provide a more complete characterization of the reference system than the implicit variants. Within each of these variant classes, the S-system representation is shown to be more mathematically tractable and accurate than the generalized mass action representation. The results allow one to make clear distinctions among the variant theories.
\end{abstract}

\section{INTRODUCTION}

It has become increasingly clear that a systematic methodology for dealing with complex biochemical systems must be developed if we are to

* Material in this paper was presented at the 6th International Conference on Mathematical Modelling, St. Louis, Missouri, 4-7 August 1987, and the 1st IFAC Symposium on Modelling and Control in Biomedical Systems, Venice, Italy, 6-9 April 1988.

${ }^{\dagger}$ Present address: Departament de Ciencies Mediques Basiques Biostadistica, Facultat de Medicina, Estudi General de Lleida, Universitat de Barcelona, 25007-LLEIDA, Spain. 
integrate the burgeoning results of studies at the molecular level with a mature, predictive understanding of the behavior of intact biochemical systems. For example, see [27] for discussion of the background and issues.

This awareness has recently focused attention on various approaches to this problem. Unfortunately, the result has been a great deal of confusion as to what different approaches do exist and how effective they are in dealing with realistic biochemical systems [e.g., see the recent Discussion Forum in Trends Biochem. Sci. 12:5-14 and 216-224 (1987)]. The confusion results from several factors. (1) A number of new approaches have been proposed during the past 20 years. (2) There has been a nearly total lack of systematic comparison of new approaches with existing approaches. (3) This has resulted in separate literatures with few cross-references between them. (4) There has been a proliferation of notation and terminology, even when standard notation and terminology already existed. (5) The specific implications of the new approaches have not been deduced and tested against actual systems, except in the simplest cases. This confusion could be eliminated if the alternative approaches were applied to the same examples and a complete and rigorous analysis were performed in each instance. This is the approach that will be taken in this paper.

The reference system in Figure 1 has been completely specified elsewhere (in particular, see Figures 2-4 and Tables 3-5 in Part I [39]) and provides an ideal reference for comparing alternative theories. ${ }^{1}$ This reference system includes many of the simple features previously assumcd by other investigators; it also includes enzyme-enzyme interactions, which are well documented but have not been included explicitly or even implicitly in most theories of intact biochemical systems. For more detailed consideration of the reference system, see the preceding paper [39].

The newer theories that have been proposed for dealing with complex biochemical systems, and that are in need of comparison, are all based on the same underlying power-law formalism $[36,37,39]$. Within this formalism there are a number of well-recognized variants that differ with regard to the degree of aggregation assumed when writing the kinetic equations for the system (reviewed in Voit and Savageau [46]). Biochemical systems theory (BST) has emphasized the S-system variant (named for its ability to capture

\footnotetext{
${ }^{1}$ The reference system was selected to operate far from thermodynamic equilibrium so that comparisons could be made among biochemical systems theory (BST), flux-oriented theory (FOT), and metabolic control theory (MCT). If the system is selected to operate around equilibrium, such comparisons cannot be made because FOT and MCT as presently formulated do not apply at equilibrium. A detailed treatment of alternative strategies within BST for representing system behavior around equilibrium can be found in Sorribas and Savageau [40].
} 


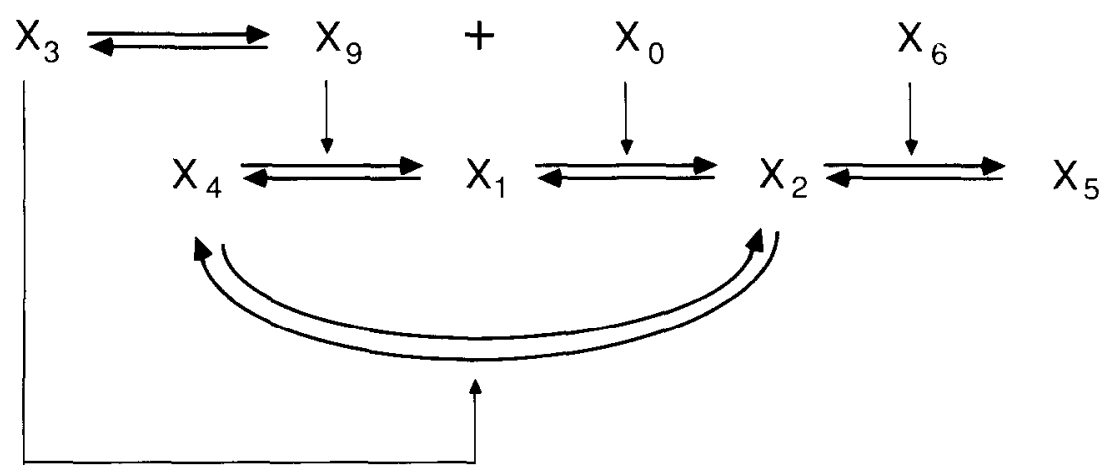

FIG. 1. Reference system that involves enzyme-enzyme interactions and channeling of metabolic flux. See Part I [39] for a detailed characterization.

the essential synergistic and saturable properties of biological processes), in which aggregation is at the level of net synthesis and net degradation for each dependent concentration variable in the system [19-24]. Numerous advantages have been documented for this variant within BST (see [36], [37], [46]).

Another common variant within BST involves the generalized mass action (GMA) representation, in which aggregation at the level of individual enzyme-catalyzed reactions is assumed [19, 36, 46]. This variant lacks the many advantages associated with the S-system variant and therefore has not been as extensively developed within BST.

Predictions based on the S-system variant within BST have been exhaustively compared with the empirically determined behavior of the reference system in Part I of this series [39]. We shall now analyze the same reference system in two different ways. First, we carry out the analysis using the explicit GMA variant of BST, which makes explicit use of the power-law formalism. Second, we present the implicit GMA variant of BST, which also is based on the power-law formalism but is perhaps less obvious. In each instance we demonstrate the range of predictions that are possible with the variant theories.

The results of detailed comparisons in this and the previous paper, using the reference system in Figure 1 as the standard, show (1) that the MCT variant is a special case of the implicit generalized mass action variant of BST, (2) that the flux-oriented theory (FOT) variant, when suitably generalized, is logically equivalent to the explicit generalized mass action variant within BST, and (3) that the explicit S-system variant is a more comprehensive and inclusive theory than the other variants, including the implicit S-system variant. 


\section{ANALYSIS USING AN EXPLICIT VARIANT OF BST: THE EXPLICIT GMA REPRESENTATION WITHIN THE POWER-LAW FORMALISM}

The approach we have referred to as FOT is based explicitly upon the power-law formalism, aggregation at the level of individual enzyme-catalyzed reactions, and the assumption of independence of enzyme levels and activities in the system [2-4]. However, in contrast with the explicit S-system variant in BST [20,39], there is no explicit steady-state solution in symbolic form that gives the systemic behavior directly in FOT. The answer to the question of how the systemic behavior of concentrations or fluxes is related to the properties of the components in the system (i.e., the parameters of the underlying representation) is obtained by differentiating the steady-state equations and solving the resulting set of linear algebraic equations (e.g., see [4]).

As a prerequisite for quantitative analysis of the intact system, we first describe the mathematical representations used in FOT and in the explicit GMA variant of BST. The explicit GMA representation, which includes FOT as a special case, is then used for the subsequent analysis because it provides a more systematic representation, allows direct comparisons with other theories in the power-law formalism, and illustrates the full potential of this class of variants within BST.

\subsection{MATHEMATICAL REPRESENTATION}

Flux-Oriented Theory (FOT). Crabtree and Newsholme [2] started with the power-law formalism as their representation of the underlying kinetics of individual reactions. For example, they wrote equations of the form

$$
v_{2}=\lambda[S]^{s_{i(s)}^{\mathrm{E}_{2}}}[P]^{s_{i(p)}^{\mathrm{E}_{2}}},
$$

where $s_{i(s)}^{\mathrm{E}_{2}}$ is called the "intrinsic sensitivity" of enzyme $\mathrm{E}_{2}$ in response to small changes in the concentration of substrate $S$, while all other metabolite concentrations are held constant. These exponents are identical to the kinetic orders of conventional chemical and biochemical kinetics, as pointed out by Savageau [20]. Although these equations are formally equivalent to those derived in BST, the way in which they are obtained represents a different level of analysis. In the original development of the power-law formalism, such equations are the result of a Taylor series expansion in a logarithmic space. Thus, the development is set within a rigorously defined mathematical context in which necessary and sufficient conditions for the validity of the 
representation exist, formulas for specifying the accuracy of the representation are known, and all the resulting parameters have a well-defined meaning. In the case of FOT, the equations are obtained by assuming constant relative variation between metabolite concentration and enzymatic rate. Integration produces the power-law function in which the exponent is the constant relative variation and the multiplicative constant is simply identified as an integration constant with no specific meaning; in fact, the same symbol $\lambda$ is used to represent two different quantities [2]. Given this difference in level of approach, it is not surprising that the accuracy of these equations is never addressed specifically in FOT.

In recent papers (e.g., [4]), these authors have replaced this notation so that the above equation would now read

$$
J_{a}=E_{1} S^{\alpha} P^{\beta},
$$

which they express in terms of the relative (or logarithmic) differentials as

$$
\stackrel{r}{J_{a}}=\stackrel{r}{E_{1}}+\alpha \stackrel{r}{S}+\beta \stackrel{r}{P}
$$

where $J_{a}=\partial J_{a} / J_{a}$, etc.

The mathematical representation of the system is then built by means of $r$ equations of this form, ${ }^{2}$ one for each reaction in the system, and $b$ equations representing Kirchhoff's flux law applied at each of the $b$ branch points in the system. The latter are of the form

$$
J_{a}+J_{b}+J_{c}+\cdots+J_{k}=0
$$

which Crabtree and Newsholme [4] write in terms of logarithmic differentials as

$$
J_{a} J_{a}^{r}+J_{b} \dot{J}_{b}+J_{c} \dot{J}_{c}^{r}+\cdots+J_{k} \dot{J}_{k}=0
$$

\footnotetext{
${ }^{2}$ In the explicit GMA variant within BST, the symbol $r$ represents a dummy index that ranges over the reactions in the system. Crabtree and Newsholme use the symbol $r$ to represent both the relative (or logarithmic) differential and the number of reactions in the system, but the meaning is usually clear from the context.
} 
where the algebraic sum is over all fluxes entering $(J>0)$ and leaving $(J<0)$ the branch point in question at steady state. Equations (1) and (2) represent $r+b$ linear equations in $r-(m-b)$ independent fluxes plus $m$ independent variables $\left(J_{a}, J_{b}, \ldots, S, P, \ldots\right)$, and these can be solved by any number of conventional methods, provided the equations are consistent and the determinant of the system in nonzero (e.g., see [27]).

Although Crabtree and Newsholme now arrange their equations in matrix-like arrays [4], they have not adopted the conventional matrix notation involving a single symbol with double subscripts, and hence fail to realize many of the advantages that have led to the selection of this convention. Instead, they have introduced the following notational convention: fluxes are represented by $J$ with a letter subscript in the sequence $a, b, c \ldots$; enzyme concentrations are represented by $E$ with a numerical subscript in the sequence $1,2,3, \ldots$; kinetic orders are represented by lowercase Greek letters in alphabetic sequence $\alpha, \beta, \gamma, \ldots$; and metabolite concentrations are represented in no particular sequence as capital Roman letters. This convention is not easy to use for large systems. Hence, for ease in making comparisons, we shall convert this to a more systematic notation in the following subsection.

In the notation of FOT the reference system in Figure 1 might be written

$$
\begin{aligned}
& J_{a}^{r}=E_{1}+\alpha \stackrel{r}{S}+\beta \dot{I}, \quad J_{a}^{r}=E_{4}^{r}+\gamma I^{r}+\delta \dot{B}, \\
& \vec{J}_{b}=\stackrel{r}{E_{2}}+\epsilon \stackrel{r}{S}+\zeta \dot{\zeta}, \quad \stackrel{r}{J}_{c}=\dot{r}_{3}+\eta \stackrel{r}{B}+\theta \stackrel{r}{P}, \\
& J_{a} \stackrel{r}{J_{a}}+J_{b} \stackrel{r}{J_{b}}-J_{c} \stackrel{r}{J}_{c}=0
\end{aligned}
$$

where substrate $\mathbf{S}=\mathrm{X}_{4}$, intermediate $\mathrm{I}=\mathrm{X}_{1}$, branch-point metabolite $\mathrm{B}=\mathrm{X}_{2}$, product $\mathrm{P}=\mathrm{X}_{5}, \mathrm{E}_{1}=\mathrm{X}_{9}, \mathrm{E}_{2}=\mathrm{X}_{3}, \mathrm{E}_{3}=\mathrm{X}_{6}, \mathrm{E}_{4}=\mathrm{X}_{0}, J_{a}=v_{41}$ $\left(=J_{d}=v_{12}\right), J_{b}=v_{42}, J_{c}=v_{25}, \alpha=g_{14}, \beta=g_{11}, \gamma=g_{211}, \delta=g_{221}, \epsilon=g_{242}$, $\zeta=g_{222}, \eta=h_{22}, \theta=h_{25}$ (see Figure 1 and the following subsection for the corresponding notation in BST). These equations can be solved for $J_{a}, f_{b}$, $J_{c}, \dot{I}$, and $\stackrel{B}{B}$ in terms of the kinetic orders $\alpha, \beta, \ldots, \theta$, the fluxes $J_{a}, J_{b}, J_{c}$, and the relative differentials $\stackrel{E}{E}_{1}, \mathscr{E}_{2}, E_{3}, \mathscr{E}_{4}, \stackrel{S}{S}$, and $\stackrel{r}{P}$.

The $E_{i}$ are not independent, as required in FOT. However, one can make the appropriate generalization of FOT by introducing the constraint relations in the power-law formalism for the total enzyme concentrations $\{3,20$, $27,28,36]$. When this procedure is followed, FOT becomes nearly identical to the explicit GMA variant within BST. To make this point clear, and to illustrate the full potential of this explicit variant, we shall present the analysis of the reference system according to the more general explicit GMA variant. 
Generalized Mass Action (GMA). As is the case in BST, one begins with the equations for the conservation of mass and the constraints on total enzyme concentration. ${ }^{3}$

$$
\begin{aligned}
\frac{d X_{1}}{d t} & =v_{41}-v_{12}, \\
\frac{d X_{2}}{d t} & =v_{12}+v_{42}-v_{25}, \\
\frac{d X_{3}}{d t} & =v_{03}-v_{30}, \\
X_{9} & =X_{7}-X_{3}, \\
X_{0} & =X_{8}-X_{3},
\end{aligned}
$$

where $v_{i j}$ is the rate of utilization of $\mathrm{X}_{i}$ for the synthesis of $\mathrm{X}_{j}$ [20]. As a preliminary step to writing the power-law equations that constitute the explicit GMA representation, Eqs. (3)-(7) are written.

$$
\begin{aligned}
& \frac{d X_{1}}{d t}=V_{1}-V_{-1}, \quad \frac{d X_{2}}{d t}=V_{21}+V_{22}-V_{-2}, \quad \frac{d X_{3}}{d t}=V_{3}-V_{-3} \\
& X_{9}=X_{7}-X_{3}, \quad X_{0}=X_{8}-X_{3} \text {, }
\end{aligned}
$$

where $V_{i}$ and $V_{-i}$ represent the net rates of synthesis and degradation of $\mathrm{X}_{i}$, and $V_{21}$ and $V_{22}$ represent the two rates of synthesis of $\mathrm{X}_{2}$ that constitute $V_{2}$, the net rate of synthesis of $X_{2}$. Hence, in this case there is no aggregation into rates for net synthesis and net degradation. This difference between the GMA variant and the S-system variant used in the previous paper affects only the representations of the net rate of synthesis for $\mathrm{X}_{2}$; all other net rates for synthesis and degradation in the system are unchanged. Hence, the conventional notation for the S-system variant within BST is valid for the GMA variant with one difference; when there is more than one reaction contributing to the net synthesis (or net degradation) of a given metabolite $X_{i}$, an additional subscript is added to identify unambiguously the individual reactions involved [35].

Each rate law and constraint relationship then is represented by an appropriate product of power-law functions, with one power law for each variable that directly influences the rate law or constraint in question $[20,27$, $28,44]$. Thus, for a system consisting of $m$ independent and $n$ dependent

\footnotetext{
${ }^{3}$ These equations are simply the rate laws and Kirchhoff's flux equations written in one of the standard forms (the "node equations") found in all areas of network analysis. See [41 (Chap. 3)] and references therein for abundant information on these topics. One should note that the equations in FOT contain the same information but in a more redundant form, as we shall see.
} 
concentration variables, the rate laws and constraints are written

$$
V_{i r}=\alpha_{i r} \prod_{j=1}^{n+m} X_{j}^{g_{i j r}}, \quad V_{-i r}=\beta_{i r} \prod_{j=1}^{n+m} X_{j}^{h_{i j r}},
$$

and

$$
X_{i}=\gamma_{i} \prod_{j=1}^{n+m} X_{j}^{f_{i j}}
$$

The multiplicative and exponential parameters are referred to as rate constants and kinetic orders, respectively. (For details see Sorribas and Savageau [39].) It should be recalled that the final $r$ subscript is dropped when there is only a single reaction contributing to the next flux for synthesis or degradation (see footnote 2).

For the specific mechanism represented in Figure 1, these equations can be written by inspection as

$$
\begin{aligned}
\frac{d X_{1}}{d t} & =\alpha_{1} X_{1}^{g_{11}} X_{4}^{g_{14}} X_{9}^{g_{19}}-\alpha_{21} X_{1}^{g_{211}} X_{2}^{g_{221}} X_{0}^{g_{201}} \\
\frac{d X_{2}}{d t} & =\alpha_{21} X_{1}^{g_{211}} X_{2}^{g_{221}} X_{0}^{g_{201}}+\alpha_{22} X_{2}^{g_{222}} X_{3}^{g_{232}} X_{4}^{g_{240}}-\beta_{2} X_{2}^{h_{22}} X_{5}^{h_{25}} X_{6}^{h_{26}} \\
\frac{d X_{3}}{d t} & =\alpha_{3} X_{9}^{g_{39}} X_{0}^{g_{30}}-\beta_{3} X_{3}^{h_{33}} \\
X_{9} & =\gamma_{9} X_{3}^{f_{93}} X_{5}^{f_{97}} \\
X_{0} & =\gamma_{0} X_{3}^{f_{03}} X_{8}^{f 08}
\end{aligned}
$$

There are two reactions producing $X_{2}$, hence the additional subscript to distinguish between them. The net rate of utilization of $X_{1}$ is identical to the net rate of production of $X_{2}$ by the first of these two reactions, so the symbols representing net degradation of $\mathrm{X}_{1}$ are replaced by the equivalent symbols in the first term of Eq. (9) for synthesis of $X_{2}[20,27]$.

The enzyme concentrations $X_{9}$ and $X_{0}$ are not independent variables, so they are replaced by using the constraints on total enzyme concentration [Eqs. (11) and (12)]. The final equations can then be written.

$$
\begin{aligned}
\frac{d X_{1}}{d t}= & \alpha_{1}^{\prime} X_{1}^{g_{11}} X_{3}^{g_{13}} X_{4}^{g_{14}} X_{7}^{g_{11}}-\alpha_{21}^{\prime} X_{1}^{g_{211}} X_{2}^{g_{221}} X_{3}^{g_{231}} X_{8}^{g_{281}}, \\
\frac{d X_{2}}{d t}= & \alpha_{21}^{\prime} X_{1}^{g_{211}} X_{2}^{g_{221}} X_{3}^{g_{231}} X_{8}^{g_{281}}+\alpha_{22} X_{2}^{g_{222}} X_{3}^{g_{232}} X_{4}^{g_{242}} \\
& -\beta_{2} X_{2}^{h_{22}} X_{5}^{h_{25}} X_{6}^{h_{26}} \\
\frac{d X_{3}}{d t}= & \alpha_{3}^{\prime} X_{3}^{g_{33}} X_{7}^{g_{37}} X_{8}^{g_{38}}-\beta_{3} X_{3}^{h_{33}}
\end{aligned}
$$


where

$$
\begin{aligned}
& \alpha_{1}^{\prime}=\alpha_{1} \gamma_{9}^{g_{19}}, \quad \alpha_{21}^{\prime}-\alpha_{21} \gamma_{0}^{g_{201}}, \quad \alpha_{3}^{\prime}-\alpha_{3} \gamma_{9}^{g_{39}} \gamma_{0}^{g_{30}}, \\
& g_{13}=g_{19} f_{93}, \quad g_{17}=g_{19} f_{97}, \quad g_{231}=g_{201} f_{03}, \quad g_{281}=g_{201} f_{08}, \\
& g_{33}=g_{39} f_{93}+g_{30} f_{03}, \quad g_{37}=g_{39} f_{97}, \quad g_{38}=g_{30} f_{08} .
\end{aligned}
$$

The parameters in this explicit variant are determined by either of the two methods described in Part I [39] for determining the parameters in the explicit S-system variant. By using information obtained from the empirical characterization of the reference system (see Figure 2 in Part I) one can estimate the parameter values and write the corresponding equations in numerical form.

$$
\begin{aligned}
\frac{d X_{1}}{d t}= & 2.44 \times 10^{-2} X_{1}^{-0.533} X_{3}^{-1.21} X_{4}^{1.07} X_{7}^{2.21}-0.315 X_{1}^{2.57} X_{2}^{-2.14} X_{3}^{-2.71} X_{8}^{3.71} \\
\frac{d X_{2}}{d t}= & 0.315 X_{1}^{2.57} X_{2}^{-2.14} X_{3}^{-2.71} X_{8}^{3.71}+1.52 X_{2}^{-0.321} X_{3}^{1.00} X_{4}^{0.400} \\
& -0.722 X_{2}^{0.568} X_{5}^{-0.0947} X_{6}^{1.00} \\
\frac{d X_{3}}{d t}= & 7.52 \times 10^{-3} X_{3}^{-3.92} X_{7}^{2.21} X_{8}^{3.71}-X_{3}^{1.00}
\end{aligned}
$$

It is instructive to compare the enumeration of equations for the explicit GMA variant within BST, which is the standard enumeration in well-developed network theories, to that in FOT. In general, the combination of rate laws and Kirchhoff's flux laws generates $r+n$ equations in $r+n$ variables, where $r$ is the number of reactions and $n$ is the number of dependent concentrations. Crabtree and Newsholme [4] define $b$ as the number of branch points in the system and have reduced the above numbers to $r+b$ equations in $r+b$ unknowns by using Kirchhoff's flux equations at the simpler, nonbranching, nodes of the system to eliminate one of a pair of equivalent flux variables. These numbers are reduced still further - to the minimum, $n$ equations in $n$ unknowns-in the explicit GMA variant within BST by substituting the appropriate rate laws into Kirchhoff's flux equations for each dependent concentration variable. This is a standard technique from network theory (see footnote 3 ).

For the reference system in Figure 1, one may consider $r=6, n=3$, and $b=1$ if the protein association and dissociation are treated as two irreversible processes. The full collection of rate laws plus Kirchhoff's node equations then generates a ninth-order system of simultaneous equations. The partial reduction given by Crabtree and Newsholme [4] yields a seventh-order system, and the fully reduced formulation in the explicit GMA variant within BST involves only a third-order system. Alternatively, one can 
assume equilibrium for the association/dissociation and eliminate these processes from consideration in steady state, and then the corresponding numbers become $r=4, n=2$, and $b=1$. The full system is now sixth-order, the partially reduced system in FOT is now fifth-order, and the fully reduced system in the explicit GMA variant is second-order. Thus, the explicit GMA variant within BST, when compared with the special case of FOT, uses a more efficient reduction of the initial $r+n$ equations and provides a more systematic representation for analysis of the system.

\subsection{BEHAVIOR OF THE CONCENTRATIONS IN STEADY STATE}

As discussed in Part I [39], the systemic behavior of a complex biochemical system is completely characterized within the context of a given model when one can predict the response of any dependent variable to change in each of the independent variables and each of the parameters of the underlying component processes. The starting point for such an analysis in the explicit GMA variant within BST is the characterization of the steady state. In a steady state the time derivatives in Eqs. (13)-(15) are equal to zero, and we have

$$
\begin{aligned}
& 0=\alpha_{1}^{\prime} X_{1}^{g_{11}} X_{3}^{g_{13}} X_{4}^{g_{14}} X_{9}^{g_{11}}-\alpha_{21}^{\prime} X_{1}^{g_{211}} X_{2}^{g_{221}} X_{\xi}^{g_{231}} X_{8}^{g_{281}} \\
& 0=\alpha_{21}^{\prime} X_{1}^{g_{211}} X_{2}^{g_{221}} X_{3}^{g_{231}} X_{8}^{g_{281}}+\alpha_{22} X_{2}^{g_{222}} X_{3}^{g_{232}} X_{4}^{g_{242}}-\beta_{2} X_{2}^{h_{22}} X_{5}^{h_{25}} X_{6}^{h_{26}} \\
& 0=\alpha_{3}^{\prime} X_{3}^{g_{33}} X_{7}^{g_{37}} X_{8}^{g_{38}}-\beta_{3} X_{3}^{h_{33}}
\end{aligned}
$$

In general, there is no explicit symbolic solution for this set of nonlinear algebraic equations [36], so the analysis cannot be as simple and direct as it was in the case of the explicit S-system variant.

Logarithmic Gains. To determine the effect of a change in one independent concentration on the steady state of the system, one differentiates each of the steady-state equations above with respect to one of the independent variables, say $X_{6}$, multiplies through by the same independent variable and divides through by the net flux, and evaluates at the steady state. In this way one obtains a set of simultaneous linear equations in the logarithmic gains $L_{16}, L_{26}$, and $L_{36}$, where $L_{i k}=\left(\partial X_{i} / \partial X_{k}\right)\left(X_{k} / X_{i}\right){ }^{4}$ [Logarithmic gains and parameter sensitivities (see below) are both referred to as "net sensitivities" in FOT and "control coefficients" in MCT.] For example, when this

\footnotetext{
${ }^{4}$ For discussion of the rationale behind this convention and of the need to distinguish clearly the responses to change in independent variables from those in the parameters of the system, see [23], [36], [37], and [39].
} 
procedure is applied to Eq. (16), one obtains

$$
\begin{gathered}
g_{11} v_{41} \frac{\partial X_{1}}{\partial X_{6}}\left(\frac{X_{6}}{X_{1}}\right)+g_{13} v_{41} \frac{\partial X_{3}}{\partial X_{6}}\left(\frac{X_{6}}{X_{3}}\right)-g_{211} v_{12} \frac{\partial X_{1}}{\partial X_{6}}\left(\frac{X_{6}}{X_{1}}\right) \\
-g_{221} v_{12} \frac{\partial X_{2}}{\partial X_{6}}\left(\frac{X_{6}}{X_{2}}\right)-g_{231} v_{12} \frac{\partial X_{3}}{\partial X_{6}}\left(\frac{X_{6}}{X_{3}}\right)=0 .
\end{gathered}
$$

By noting the definition of logarithmic gain given above, and the identity of $v_{41}$ and $v_{12}$ in steady state (i.e., $v_{41}=v_{12} \neq 0$ ), one can write this equation as

$$
\left(g_{11}-g_{211}\right) L_{16}-g_{221} L_{26}+\left(g_{13}-g_{231}\right) L_{36}=0 .
$$

The other two equations follow in a similar fashion:

$$
\begin{gathered}
g_{211} \frac{v_{12}}{v_{25}} L_{16}+\left(g_{221} \frac{v_{12}}{v_{25}}+g_{222} \frac{v_{42}}{v_{25}}-h_{22}\right) L_{26}+\left(g_{231} \frac{v_{12}}{v_{25}}+g_{232} \frac{v_{42}}{v_{25}}\right) L_{36}=h_{26}, \\
a_{33} L_{36}=0 .
\end{gathered}
$$

These linear equations can be solved for $L_{16}, L_{26}$, and $L_{36}$ provided the determinant of the coefficients on the left-hand side is nonzero. Note that this determinant is identical to the $n \times n$ system determinant in the explicit S-system variant (Eq. (23) in Sorribas and Savageau [39]) because in the power-law formalism the kinetic orders for the net rate law are simply the average of the corresponding kinetic orders for the individual rate laws, weighted according to the fraction of net flux contributed by the individual fluxes. That is,

$$
g_{21}=g_{211} \frac{v_{12}}{v_{25}}, \quad g_{22}=g_{221} \frac{v_{12}}{v_{25}}+g_{222} \frac{v_{42}}{v_{25}}, \quad g_{23}^{\prime}=g_{231} \frac{v_{12}}{v_{25}}+g_{232} \frac{v_{42}}{v_{25}},
$$

by the definition of kinetic order $[20,36]$.

There are 12 more logarithmic gains for the reference system-three for each of the four remaining independent variables. The above procedure must therefore be repeated for each of these four independent variables. We shall not go through the details. One obtains the same results as given earlier for the explicit S-system variant within BST. Thus, the five solutions for the third-order sets of linear algebraic equations involve inversion of the same system matrix of kinetic orders. Given that the vector of constants changes in association with the choice of independent variable, it follows that the explicit S-system variant, which involves inversion of the same system matrix, must provide the same information, but we believe it is provided in a more systematically organized form [39]. 
In either case, the difficult part of the solution, namely inversion of the system matrix, is considerably simplified by using the fully reduced form of the system equations found in the GM $\Lambda$ variant rather than the partially reduced form found in FOT, as was pointed out in Section 2.1.

It is also important to emphasize here that the identity of results with thesc alternative representations holds only at a single point - the steady state. The alternative representations differ elsewhere, in general, and the relative accuracy with which they represent the reference system will differ.

Rate-Constant Sensitivities. To determine the response of a dependent variable to change in one of the rate constants of the system, one can differentiate the steady-state equations [e.g., Eqs. (16)-(18)] with respect to a rate-constant parameter (e.g., $\beta_{3}$ ), multiply through by that parameter and divide through by the net flux, and evaluate the results at the steady-state operating point. The result is a set of linear algebraic equations in the sensitivities $S\left(X_{1}, \beta_{3}\right), S\left(X_{2}, \beta_{3}\right)$, and $S\left(X_{3}, \beta_{3}\right)$, where $S\left(X_{i}, \beta_{j}\right)=$ $\left(\partial X_{i} / \partial \beta_{j}\right)\left(\beta_{j} / X_{i}\right)$ (see footnote 4$)$.

$$
\begin{gathered}
\left(g_{11}-g_{211}\right) S\left(X_{1}, \beta_{3}\right)-g_{221} S\left(X_{2}, \beta_{3}\right)+\left(g_{13}-g_{231}\right) S\left(X_{3}, \beta_{3}\right)=0 \\
g_{211} \frac{v_{12}}{v_{25}} S\left(X_{1}, \beta_{3}\right)+\left(g_{221} \frac{v_{12}}{v_{25}}+g_{222} \frac{v_{42}}{v_{25}}-h_{22}\right) S\left(X_{2}, \beta_{3}\right) \\
+\left(g_{231} \frac{v_{12}}{v_{25}}+g_{232} \frac{v_{42}}{v_{25}}\right) S\left(X_{3}, \beta_{3}\right)=0 \\
a_{33} S\left(X_{1}, \beta_{3}\right)=1 .
\end{gathered}
$$

Note that this system of equations has the same determinant as Eqs. (19)-(21), only the right-hand sides differ.

To obtain the 15 additional sensitivities with respect to the five remaining rate constants of our reference system, the above procedure must be repeated for each of these five rate constants. In cach casc, onc obtains the same matrix of coefficients on the left-hand side, but different right-hand sides of these equations. This procedure yields the same results that were obtained directly using the explicit S-system variant within BST.

There are no comparable results in FOT because rate-constant parameters have not been clearly distinguished in FOT; it is clear that this could be done by generalizing FOT and one would obtain results identical to those presented above.

Kinetic-Order Sensitivities. In a similar fashion, one can determine the systemic responses to change in the kinetic orders of the system by differentiating the steady-state equations with respect to a kinetic-order parameter 
(e.g., $g_{232}$ ), multiplying through by this parameter and dividing by the net flux, and evaluating the results at the steady-state operating point. The result is a set of linear algebraic equations in the sensitivities $S\left(X_{1}, g_{232}\right)$, $S\left(X_{2}, g_{232}\right)$, and $S\left(X_{3}, g_{232}\right)$, where $S\left(X_{i}, g_{p k s}\right)=\left(\partial X_{i} / \partial g_{p k s}\right)\left(g_{p k s} / X_{i}\right)$.

$$
\begin{gathered}
\left(g_{11}-g_{211}\right) S\left(X_{1}, g_{232}\right)-g_{221} S\left(X_{2}, g_{232}\right)+\left(g_{13}-g_{231}\right) S\left(X_{3}, g_{232}\right)=0 \\
g_{211} \frac{v_{12}}{v_{25}} S\left(X_{1}, g_{232}\right)+\left(g_{221} \frac{v_{12}}{v_{25}}+g_{222} \frac{v_{42}}{v_{25}}-h_{22}\right) S\left(X_{2}, g_{232}\right) \\
+\left(g_{231} \frac{v_{12}}{v_{25}}+g_{232} \frac{v_{42}}{v_{25}}\right) S\left(X_{3}, g_{232}\right)=-y_{3} g_{232} \frac{v_{42}}{v_{25}} \\
a_{33} S\left(X_{1}, g_{232}\right)=0
\end{gathered}
$$

Note that this system of equations also has the same determinant as Eqs. (19)-(21), only the right-hand sides differ. For this class of parameters the solution must be repeated another 17 times in order to obtain the complete set of parameter sensitivities.

Again, there are no comparable results in FOT; it is clear that such predictions could be made and that one would obtain results identical to those presented above using the explicit GMA variant within BST.

In all, a similar set of equations must be formulated and solved 29 times. These equations can be organized into a single matrix equation, which exhibits the relationship to the equations of the explicit S-system variant. In any case, one obtains the same results that were obtained directly using the explicit S-system variant within BST.

\subsection{BEHAVIOR OF THE FLUXES IN STEADY STATE}

In the power-law formalism the flux through any given process is obtained by a secondary calculation once the values of the dependent concentrations have been determined, i.e.,

$$
V_{i r}=\alpha_{i r}^{\prime} \prod_{j=1}^{8} X_{j}^{g_{i t r}}, \quad i=1,2,3 ; r=1,2
$$

From this equation, one also can predict the behavior of the fluxes at steady state in response to changes in the independent variables and parameters of the system. 
Logarithmic Gains. The logarithmic derivative of such a function with respect to an independent variable gives

$$
\begin{aligned}
L\left(V_{i r}, X_{k}\right) & =\frac{\partial\left(\log V_{i r}\right)}{\partial\left(\log X_{k}\right)} \\
& =g_{i k r}+\sum_{j=1}^{3} g_{i j r} L\left(X_{j}, X_{k}\right), \quad i=1,2,3 ; k=4, \ldots, 8 ; r=1,2 .
\end{aligned}
$$

Rate-Constant Sensitivities. The logarithmic derivative with respect to a rate-constant parameter yields

$$
S\left(V_{i r}, \alpha_{k s}^{\prime}\right)=\delta_{i k} \delta_{r s}+\sum_{j=1}^{3} g_{i j r} S\left(X_{j}, \alpha_{k s}^{\prime}\right)
$$

or

$$
S\left(V_{i r}, \beta_{k s}\right)=\sum_{j=1}^{3} g_{i j r} S\left(X_{j}, \beta_{k s}\right), \quad i, k=1,2,3 ; r, s=1,2,
$$

where $\delta_{i k}$ is the Kronecker delta symbol, which is equal to 1 when $i=k$ and 0 otherwise.

Kinetic-Order Sensitivities. Similarly, the logarithmic derivative with respect to a kinetic-order parameter yields

$$
S\left(V_{i r}, g_{p k s}\right)=g_{p k s} y_{p} \delta_{i p} \delta_{r s}+\sum_{j=1}^{3} g_{i j r} S\left(X_{j}, g_{p k s}\right)
$$

or

$$
S\left(V_{i r}, h_{p k s}\right)=\sum_{j=1}^{3} g_{i j r} S\left(X_{j}, h_{p k s}\right), \quad i, p=1,2,3 ; r, s=1,2 ; k=1, \ldots, 8 .
$$

Equations (30) and (32) have no Kronecker deltas because the $\beta$ and $h$ parameters do not appear in the $V_{i r}$ terms.

A set of similar relationships can be written for the degradative rate laws as well. However, in steady state,

$$
V_{-i}=V_{i}=\sum_{r} V_{i r}, \quad i=1,2,3,
$$


which shows that only three of the four fluxes represented in Figure 1 are independent of each other. Hence, we need only determine three fluxes in order to characterize the systemic behavior. We choose $V_{1}, V_{2}$, and $V_{3}$ as we did in Part I for the explicit S-system variant within BST [39]. The remaining flux $V_{22}$ is given by the difference $V_{2}-V_{1}$.

Thus, once the concentration behavior has been determined, the flux behavior follows by multiplication and addition.

\subsection{BEHAVIOR ABOUT THE NOMINAL STEADY STATE}

A critical step in establishing the validity of any model is to demonstrate that it possesses a steady state corresponding to that exhibited by the actual system. The explicit GMA variant does not permit an explicit solution for the steady-state equations, and one cannot derive an existence theorem for the steady state in the way it was done for the explicit S-system variant within BST [20,36]. This is a limitation of the GMA variant, but it can be overcome to some extent, because one can study the behavior within a local neighborhood of the steady state by means of a numerical solution to Eqs. (13)-(15). For this purpose we have employed ESSYNS, the program described in Part I. These solutions also give the local dynamic behavior of the system. As yet, there are no comparable results in FOT, although it is clear that ESSYNS can be applied in FOT and one would obtain identical results.

Another important test for any model that is to represent a real system with a stable steady state is the existence of stability in the model. The necessary conditions for local stability play an important role in evaluating alternative biochemical designs that have evolved (e.g., see Savageau [26, 27, 30]). One can determine the local stability properties of models in the explicit GMA variant by using standard techniques from linear analysis. The linearized equivalent of Eqs. (13) (15) in the local neighborhood of the steady state can be written

$$
\frac{d u_{i}}{d t}=\sum_{j=1}^{n}\left(\sum_{r} g_{i j r} \frac{V_{i r 0}}{X_{i 0}}-\sum_{r} h_{i j r} \frac{V_{-i r 0}}{X_{i 0}}\right) u_{j},
$$

where $u_{i}=\left(X_{i}-X_{i 0}\right) / X_{i 0}$ and the additional zero subscript signifies steady-state values. With this linear representation one can explore the conditions for stability of the steady state, e.g., by using the Routh criteria (e.g., see [27]). When one takes into account the meaning of the kinetic orders in the GMA and S-system variants, then one sees that

$$
\sum_{r} g_{i j r} \frac{V_{i r 0}}{V_{i 0}}-\sum_{r} h_{i j r} \frac{V_{-i r 0}}{V_{i 0}}=a_{i j}
$$


and therefore that Eq. (33) can be written as

$$
\frac{d u_{i}}{d t}=\sum_{j=1}^{n} \frac{V_{i 0}}{X_{i 0}} a_{i j} u_{j}
$$

This equation is identical to the one previously obtained for the S-system variant within BST [26], and hence the resulting conditions for stability are identical to those obtained for the S-system variant $[26,27]$.

\subsection{RESULTS}

Logarithmic Gains. The responses of the dependent variables $X_{1}, X_{2}$, and $X_{3}$ to changes in the independent variables $X_{4}, X_{5}, X_{6}, X_{7}$, and $X_{8}$ are obtained by solving sets of equations such as Eqs. (19)-(21). The responses of the flux variables $V_{1}, V_{2}$, and $V_{3}$ follow from Eq. (28). The results are summarized in Table 1. It can be seen that these results are identical to those obtained previously with the explicit S-system variant within BST (see Table 3 in Part I) and that they are in good agreement with the empirical data obtained directly from the reference system.

Parameter Sensitivities. The responses of the dependent variables to changes in the rate-constant parameters are obtained by first solving sets of equations such as Eqs. (22)-(24). The complete set of these parameter sensitivities is given in Table 2. For all the rate constants, except $\alpha_{21}^{\prime}$ and $\alpha_{22}$, the results are identical to those obtained previously with the explicit S-system variant (see Table 4 in Part I). The sensitivities with respect to

\section{TABLE 1}

Logarithmic Gains: Percentage Change in the Dependent Variables of the System in Response to a 1\% Change in an Independent Variable ${ }^{a}$

\begin{tabular}{ccccccc}
\hline \multirow{2}{*}{$\begin{array}{c}\text { Independent } \\
\text { variable }\end{array}$} & $X_{1}$ & $X_{2}$ & $X_{3}$ & $V_{1}$ & $V_{2}$ & $V_{3}$ \\
\cline { 2 - 7 }$X_{4}$ & 0.726 & 0.553 & 0.00 & 0.683 & 0.314 & 0.00 \\
$X_{5}$ & 0.0727 & 0.105 & 0.00 & -0.0387 & -0.0348 & 0.00 \\
$X_{6}$ & -0.768 & -1.11 & 0.00 & 0.410 & 0.368 & 0.00 \\
$X_{7}$ & 1.39 & 0.661 & 0.449 & 0.928 & 0.375 & 0.449 \\
$X_{8}$ & -0.440 & 0.567 & 0.754 & -0.678 & 0.322 & 0.754 \\
\hline
\end{tabular}

${ }^{a}$ Determined for the reference system, the explicit GMA variant within BST, or the implicit GMA variant within BST. 
TABLE 2

Rate-Constant Sensitivities: Percentage Change in the Dependent Variables of the System in Response to a $1 \%$ Change in a Rate Constant ${ }^{\mathrm{a}}$

\begin{tabular}{crccccc}
\hline \multirow{2}{*}{$\begin{array}{c}\text { Rate } \\
\text { constant }\end{array}$} & \multicolumn{1}{c}{$X_{1}$} & \multicolumn{1}{c}{$X_{2}$} & \multicolumn{1}{c}{$X_{3}$} & \multicolumn{1}{c}{$V_{1}$} & \multicolumn{1}{c}{$V_{2}$} & $V_{3}$ \\
\hline$\alpha_{1}^{\prime}$ & 0.450 & 0.184 & 0.00 & 0.760 & 0.105 & 0.00 \\
$\alpha_{21}^{\prime} \mathrm{b}$ & -0.296 & 0.0386 & 0.00 & 0.158 & 0.0214 & 0.00 \\
$\alpha_{22} \mathrm{~b}$ & 0.614 & 0.890 & 0.00 & -0.328 & 0.506 & 0.00 \\
$\beta_{2}$ & -0.768 & -1.11 & 0.00 & 0.410 & 0.367 & 0.00 \\
$\alpha_{3}^{\prime}$ & 0.177 & 0.115 & 0.203 & -0.340 & 0.0651 & 0.203 \\
$\beta_{3}$ & -0.177 & -0.115 & -0.203 & 0.340 & -0.0651 & 0.797 \\
\hline
\end{tabular}

${ }^{a}$ Determined for the explicit GMA variant within BST.

${ }^{b}$ The sensitivities with respect to these rate constants in the explicit GMA variant are directly related to the sensitivities with respect to $\beta_{1}^{\prime}$ and $\alpha_{2}^{\prime}$ in the explicit S-system variant (see text).

change in $\alpha_{21}^{\prime}$ and $\alpha_{22}$ in the GMA variant are directly related to those with respect to $\beta_{1}^{\prime}$ and $\alpha_{2}^{\prime}$ in the S-system variant by noting the following relationships that are a consequence of the definitions in the power-law formalism:

$$
\begin{aligned}
& S\left(X_{i}, \alpha_{21}^{\prime}\right)=S\left(X_{i}, \beta_{1}^{\prime}\right)+\frac{v_{12}}{v_{25}} S\left(X_{i}, \alpha_{2}^{\prime}\right), \\
& S\left(V_{i}, \alpha_{21}^{\prime}\right)=S\left(V_{i}, \beta_{1}^{\prime}\right)+\frac{v_{12}}{v_{25}} S\left(V_{i}, \alpha_{2}^{\prime}\right), \\
& S\left(X_{i}, \alpha_{22}\right)=\frac{v_{42}}{v_{25}} S\left(X_{i}, \alpha_{2}^{\prime}\right), \quad S\left(V_{i}, \alpha_{22}\right)=\frac{v_{42}}{v_{25}} S\left(V_{i}, \alpha_{2}^{\prime}\right), \quad i=1,2,3 .
\end{aligned}
$$

By substituting the appropriate numerical values, one sees that these two sets of parameter sensitivities are also in agreement.

The responses of the dependent variables to changes in the kinetic-order parameters are obtained by solving equations such as Eqs. (25)-(27). The complete set of these parameter sensitivities is given in Table 3. For all the kinetic orders, except $g_{211}, g_{221}, g_{231}, g_{281}, g_{222}, g_{232}$, and $g_{242}$, the results are identical to those obtained previously with the explicit S-system variant (see Table 5 of Part I). The sensitivities with respect to the kinetic orders $g_{2 j 1}$ $(j=1,2,3,8)$ and $g_{2 k 2}(k=2,3,4)$ in the generalized mass action variant are directly related to those with respect to $h_{1 j}$ and $g_{2 k}$ in the S-system variant, 
TABLE 3

Kinetic-Order Sensitivities: Percentage Change in the Dependent Variables of the System in Response to a 1\% Change in a Kinetic Order ${ }^{\text {a }}$

\begin{tabular}{lcccccc}
\hline \multirow{2}{*}{$\begin{array}{c}\text { Kinetic } \\
\text { order }\end{array}$} & $X_{1}$ & $X_{2}$ & $X_{3}$ & $V_{1}$ & $V_{2}$ & $V_{3}$ \\
\hline & -0.386 & -0.158 & 0.00 & -0.653 & -0.0899 & 0.00 \\
\hline$g_{11}$ & -1.30 & -0.534 & 0.00 & -2.20 & -0.303 & 0.00 \\
$g_{13}$ & 1.11 & 0.454 & 0.00 & 1.87 & 0.258 & 0.00 \\
$g_{14}$ & 2.98 & 1.22 & 0.00 & 5.03 & 0.694 & 0.00 \\
$g_{17}$ & -1.22 & 0.159 & 0.00 & 0.652 & 0.0900 & 0.00 \\
$g_{211} \mathrm{~b}$ & 1.45 & -0.185 & 0.00 & -0.777 & -0.107 & 0.00 \\
$g_{221} \mathrm{~b}$ & 1.92 & -0.243 & 0.00 & -1.02 & -0.139 & 0.00 \\
$g_{231} \mathrm{~b}$ & -2.98 & 0.390 & 0.00 & 1.59 & 0.220 & 0.00 \\
$g_{281} \mathrm{~b}$ & -0.454 & -0.656 & 0.00 & 0.242 & -0.374 & 0.00 \\
$g_{222} \mathrm{~b}$ & 1.47 & 2.13 & 0.00 & -0.784 & 1.21 & 0.00 \\
$g_{232}{ }_{\mathrm{b}}$ & 0.566 & 0.821 & 0.00 & -0.301 & 0.466 & 0.00 \\
$g_{242} \mathrm{~b}$ & -1.00 & -1.46 & 0.00 & 0.535 & 0.479 & 0.00 \\
$h_{22}$ & 0.0504 & 0.0731 & 0.00 & -0.0269 & -0.0241 & 0.00 \\
$h_{25}$ & -1.77 & -2.57 & 0.00 & 0.942 & 0.845 & 0.00 \\
$h_{26}$ & -1.66 & -1.08 & -1.91 & 3.19 & -0.611 & -1.91 \\
$g_{33}$ & 1.17 & 0.758 & 1.35 & -2.25 & 0.431 & 1.35 \\
$g_{37}$ & 1.78 & 1.15 & 2.04 & -3.42 & 0.654 & 2.04 \\
$g_{38}$ & -0.424 & -0.274 & -0.487 & 0.815 & -0.156 & 1.91 \\
$h_{33}$ & & &
\end{tabular}

${ }^{\text {a }}$ Determined for the explicit GMA variant within BST.

${ }^{b}$ The sensitivities with respect to these kinetic orders in the explicit GMA variant are directly related to the sensitivities with respect to $h_{11}, h_{12}, h_{13}, h_{18}, g_{21}, g_{22}, g_{23}^{\prime}, g_{24}$, and $g_{28}$ in the explicit S-system variant (see text).

again as a consequence of the definitions in the power-law formalism:

$$
\begin{aligned}
& \frac{S\left(X_{i}, g_{2 k 1}\right)}{g_{2 k 1}}=\frac{S\left(X_{i}, h_{1 k}\right)}{h_{1 k}}+\frac{v_{12}}{v_{25}}\left(\frac{S\left(X_{i}, g_{2 k}\right)}{g_{2 k}}\right) \\
& \frac{S\left(V_{i}, g_{2 k 1}\right)}{g_{2 k 1}}=\frac{S\left(V_{i}, h_{1 k}\right)}{h_{1 k}}+\frac{v_{12}}{v_{25}}\left(\frac{S\left(V_{i}, g_{2 k}\right)}{g_{2 k}}\right), \quad i=1,2,3 ; k=1,2,3,8 ; \\
& \frac{S\left(X_{i}, g_{2 k 2}\right)}{g_{2 k 2}}=\frac{v_{42}}{v_{25}}\left(\frac{S\left(X_{i}, g_{2 k}\right)}{g_{2 k}}\right) \\
& \frac{S\left(V_{i}, g_{2 k 2}\right)}{g_{2 k 2}}=\frac{v_{42}}{v_{25}}\left(\frac{S\left(V_{i}, g_{2 k}\right)}{g_{2 k}}\right), \quad i=1,2,3 ; k=2,3,4 .
\end{aligned}
$$


This is the result of partitioning the effects of change in an aggregated parameter into the effects of change in the corresponding parameters for each of the converging processes, weighted according to the fraction of flux generated by each process [37].

Thus, in all cases the steady-state results from the generalized mass action variant are equivalent to those from the S-system variant when one takes into account the above identities that are a consequence of the definitions in the power-law formalism and restricts the comparisons to the steady-state operating point. Results involving parameters that are common to both variants are identical. Results involving parameters that are unique to each of the variants are interconvertible between the variants; i.e., one can obtain these results either directly from one variant or indirectly from the other (via the above identities), and the answer will be the same.

Behavior about the Nominal Steady State. The predicted steady-state responses in the local neighborhood of the nominal operating point have been determined numerically by using ESSYNS. For relatively wide variations about the steady state, these results agree with the empirical data obtained directly from the reference system. Representative results exhibiting narrow and wide ranges of agreement are shown graphically in Figure 2. The ranges over which the predicted and actual responses agree to within $10 \%$ are summarized in Table 4 for all responses to all independent variables. The ranges for the generalized mass action variant are quite large, from a minimum of twofold to a maximum of 63 -fold with an average of 15 -fold, which is considerably greater than the $<10 \%$ range quoted by others $[2,48]$. These ranges are usually smaller than the corresponding ranges for the S-system variant (average range 20-fold), in agreement with the general conclusions obtained by an alternative procedure in Voit and Savageau [46]. As indicated in that paper, there can be a small minority of concentrations or fluxes within a system for which the generalized mass action variant will be more accurate, but even in these cases the additional accuracy is never greater than about $15 \%$ for the reference systems in Figure 1.

A typical dynamic response to a change within the local neighborhood of the steady state is shown in Figure 3. The results predicted by the GMA variant within BST $(G)$ are in reasonable agreement with the corresponding empirical data obtained directly from the reference system $(R)$. The average error between these responses is about $7 \%$. By comparison, the average error is about $5 \%$ when the response is predicted by the S-systcm variant of BST $(S)$. Similar results have been obtained for other responses (see Part I [39] for details). 


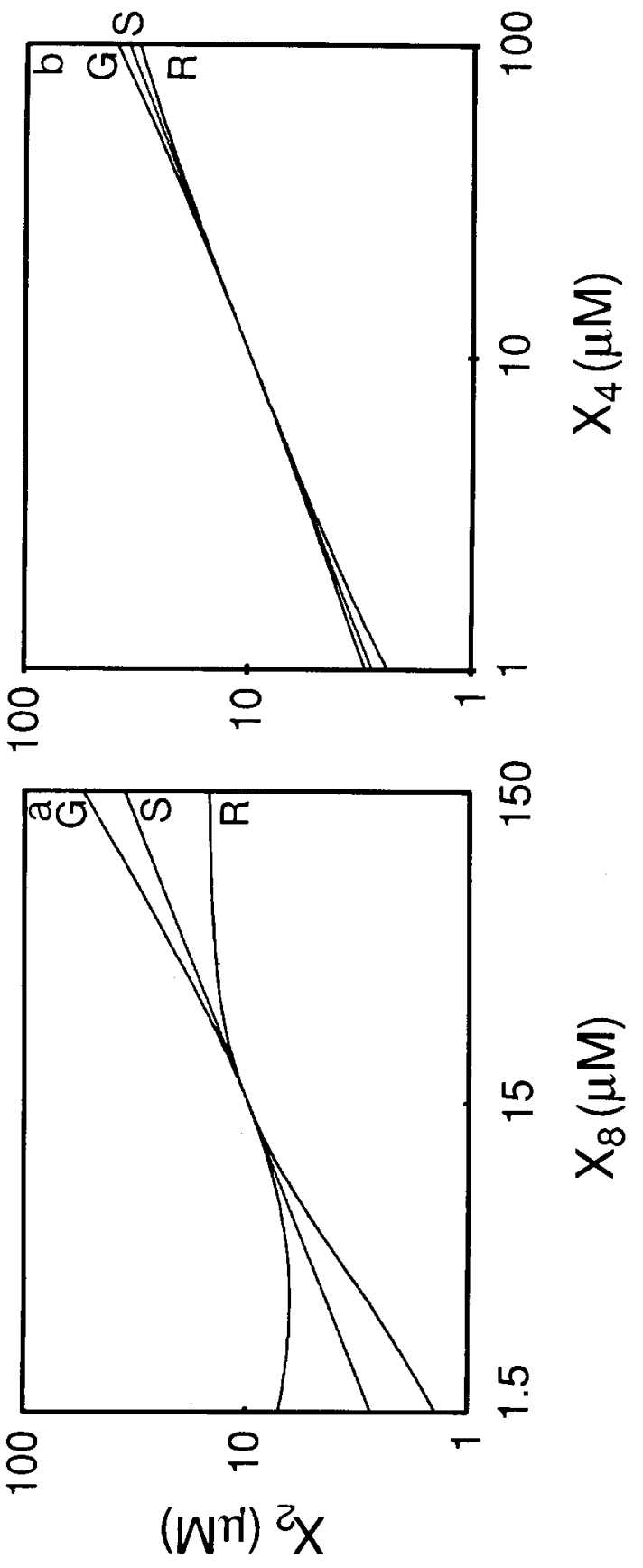

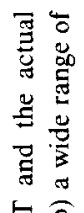

氛

苛

弄

苞

$\stackrel{5}{\circ}$

(0)

$\sum_{0}$

녈

ส

E

事

$\dot{s}$ : $\nabla$

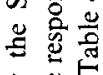

衣.

吾焉

造总

远整

농로

ङ

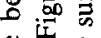

핑

공

通焉

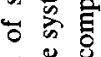

ธ

总总爮

范

ن

त है

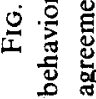


TABLE 4

Range of Concentrations Over Which the Explicit GMA Variant within BST Is Accurate ${ }^{\mathrm{a}}$

\begin{tabular}{ccccccc}
\hline \multirow{2}{*}{$\begin{array}{c}\text { Independent } \\
\text { variable }\end{array}$} & $X_{1}$ & $X_{2}$ & $X_{3}$ & $V_{1}$ & $V_{2}$ & $V_{3}^{\prime}$ \\
\cline { 2 - 7 }$X_{4}$ & $>62.7$ & 21.8 & $\infty^{\mathrm{c}}$ & 6.63 & 10.9 & $\infty$ \\
& $(>97.5)^{\mathrm{b}}$ & $(55.6)$ & $\infty$ & $(6.27)$ & $(13.6)$ & $\infty$ \\
$X_{5}$ & 48.5 & 27.1 & $\infty$ & 58.2 & 61.3 & $\infty$ \\
& $(48.5)$ & $(27.1)$ & $\infty$ & $(58.2)$ & $(61.3)$ & $\infty$ \\
$X_{6}$ & 7.06 & 8.88 & $\infty$ & 4.22 & 4.09 & $\infty$ \\
& $(7.44)$ & $(10.1)$ & $\infty$ & $(4.22)$ & $(4.06)$ & $\infty$ \\
$X_{7}$ & 4.44 & 2.59 & 3.34 & 2.17 & 3.06 & 3.34 \\
& $(4.37)$ & $(2.76)$ & $(3.34)$ & $(1.98)$ & $(3.21)$ & $(3.34)$ \\
$X_{8}$ & 3.02 & 2.65 & 4.11 & 2.01 & 3.28 & 4.11 \\
& $(2.58)$ & $(3.38)$ & $(4.11)$ & $(1.96)$ & $(4.10)$ & $(4.11)$ \\
\hline
\end{tabular}

${ }^{a}$ The range is measured by the ratio of the largest to the smallest values of the independent variable that leave the dependent variable within $10 \%$ of its actual value. The larger this range, the greater the accuracy of the representation.

${ }^{b}$ The range for the explicit S-system variant is given in parentheses for comparison (see text for discussion).

${ }^{\mathrm{c}}$ The dependent variables $X_{3}$ and $V_{3}$ are not influenced by changes in the independent variables $X_{4}, X_{5}, X_{6}$.

Summary of Results. From the results in this section it can be concluded that FOT is a special case of the explicit GMA variant within BST. By gcncralizing FOT to include (1) treatment of enzyme-enzyme interactions, (2) formulation as conventional mass balance equations, (3) explicit representation of the rate-constant parameters, (4) dynamics, and (5) parameter sensitivities, we have demonstrated that it becomes logically equivalent to the explicit GMA variant within BST. Even when the full potential of this class of explicit variants is realized, as we have seen with the GMA variant, the results are typically less accurate than those obtained with the S-system variant. The analysis also is less tractable mathematically. The results in this section show that neither FOT nor the more general explicit GMA variant within BST yields any information that is not provided by the explicit S-system variant within BST.

\section{ANALYSIS USING AN IMPLICIT VARIANT OF BST: THE IMPLICIT GMA REPRESENTATION WITHIN THE POWER-LAW FORMALISM}

In the approach referred to as metabolic control theory (MCT) there is no explicit representation of the underlying kinetic equations, and hence there is 


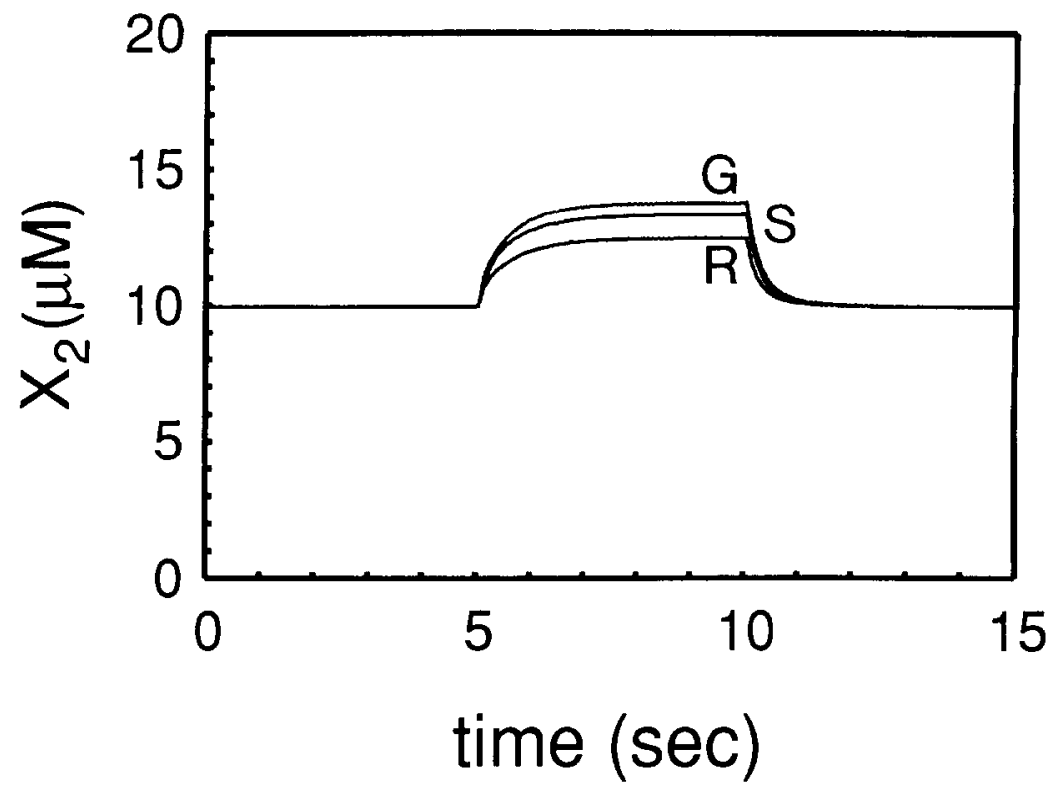

FIG. 3. Dynamic response to a change within the local neighborhood of the steady state. Before time equals $5 \mathrm{~s}$ the system is in steady state. At time equals $5 \mathrm{~s}$ the independent concentration $X_{8}$ is increased from 15 to $25 \mu \mathrm{M}$ by the addition of free enzyme $X_{0}$. At time equals $10 \mathrm{~s}$ the concentration $X_{8}$ is decreased from 25 to $15 \mu \mathrm{M}$. The response predicted by the explicit S-system (S) and the explicit GMA (G) variants within BST are contrasted with the empirical data obtained directly from the reference system (R) in Figure 1.

no explicit steady-state solution that gives the systemic behavior directly [36]. The answer to the question of how the systemic behavior of concentrations or fluxes is related to the component properties (the parameters of the underlying representation) is solved indirectly from a set of constraint relationships $[6,7,13,47]$ called summation and connectivity relationships or "theorems."

As a preliminary step to the analysis using implicit methods, we summarize the mathematical representation used in MCT and in the implicit GMA variant within BST. The implicit GMA variant, which includes MCT as a special case, is then used for subsequent analysis because it provides a valid representation of the reference system, allows direct comparisons with other theories in the power-law formalism, and illustrates the full potential of this class of implicit variants within BST. 


\subsection{MATHEMATICAL REPRESENTATION}

Metabolic Control Theory (MCT). The summation and connectivity relationships involve two types of coefficients. Those that involve changes of enzyme rate in response to independent changes in metabolite concentrations are called "elasticities" (for a review, see [15]); they are identical to the kinetic orders in chemical and biochemical kinetics [20,36].

$$
\epsilon_{\mathrm{X}_{j}}=\frac{\partial v_{i}}{\partial X_{j}}\left(\frac{X_{j}}{v_{i}}\right), \quad i=3,6,9,0 ; j=1,2
$$

Those coefficients that involve systemic changes of metabolite concentrations in response to independent changes ${ }^{5}$ in enzyme levels or activities are called "control coefficients" [15]; these are special cases of sensitivity coefficients $[22,23]$ when reactions are linear in enzyme concentration or activity $[36,37]$.

$$
C_{\mathrm{E}_{k}}^{\mathrm{X}_{j}}=\frac{\partial X_{j}}{\partial E_{k}}\left(\frac{E_{k}}{X_{j}}\right), \quad j=1,2 ; k=3,6,9,0
$$

Four types of constraints involving only the above two types of coefficients have been described: (1) flux summation [6,13], (2) flux connectivity [13], (3) concentration summation [6], and (4) concentration connectivity [47]. [For example, see Eqs. (34) and (35).] These relationships have been derived by various arguments and it has been claimed that they are entirely general (most recently by Kacser and Porteous [15]). It is shown elsewhere by Savageau et al. [37] that these are special cases of more general relationships that are mathematically identical to the well-known orthogonality properties of linear systems.

Although these relationships apply to branched pathways under appropriate restrictions, they are not sufficient to solve for the control coefficients in terms of the elasticities. To relate these two types of coefficients one must

\footnotetext{
${ }^{5}$ It is fundamental to any analysis of biochemical systems that independent and dependent variables be clearly distinguished. According to MCT, each enzyme level in the system is considered to be an independent variable, and hence one can determine control coefficients for each enzyme by varying its level experimentally [15]. This is not possible in systems for which there are enzyme-proenzyme cascades or enzyme-enzyme complexes, as has been pointed out $[31,37]$. In these types of systems, enzyme levels are dependent variables and cannot be directly manipulated.
} 
utilize another set of relationships in MCT that involve the steady-state fluxes at branch points [5, 7, 12]. [For example, see Eqs. (36) and (37).]

Focusing on these summation, connectivity, and auxiliary relationships leads to a special mathematical representation of the system. In the case of the reference system in Figure 1, an enumeration of these relationships leads to the following equations.

$$
\begin{aligned}
& C_{\mathrm{E}_{3}}^{\mathbf{X}_{i}}+C_{\mathrm{E}_{\mathrm{o}}}^{\mathbf{X}_{i}}+C_{\mathrm{E}_{y}}^{\mathbf{X}_{i}}+C_{\mathrm{E}_{0}}^{\mathbf{X}_{i}}=0 \\
& C_{\mathrm{E}_{3}}^{\mathrm{X}_{i}} \epsilon_{\mathrm{X}_{j}}^{\mathrm{E}_{3}}+C_{\mathrm{E}_{6}}^{\mathrm{E}_{i}} \epsilon_{\mathrm{X}_{j}}^{\mathrm{E}_{j}}+C_{\mathrm{E}_{9}^{\prime}}^{\mathrm{E}_{i}} \mathrm{E}_{\mathrm{X}_{j}}^{\mathrm{E}_{j}}+C_{\mathrm{E}_{0}}^{\mathrm{X}_{i} \epsilon} \epsilon_{\mathrm{X}_{j}}^{\mathrm{E}_{j}}=-\delta_{i j}, \quad i, j=1,2 ; \\
& \left(V_{\mathrm{E}_{9}} \epsilon_{\mathbf{X}_{1}}^{\mathrm{E}_{9}}-V_{\mathrm{E}_{0}} \epsilon_{\mathbf{X}_{1}^{0}}^{\mathrm{E}_{0}}\right) c_{\mathrm{E}_{k}}^{\mathrm{X}_{1}}-V_{\mathrm{E}_{0}} \epsilon_{\mathbf{X}_{2}^{0}}^{\mathrm{E}_{0}} C_{\mathrm{E}_{k}}^{\mathbf{X}_{2}}=b_{1 k}
\end{aligned}
$$

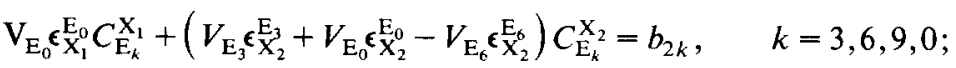

where $b_{13}=b_{16}=b_{29}=0, b_{19}=-V_{\mathrm{E}_{9}}, b_{10}=V_{\mathrm{E}_{0}}, b_{23}=-V_{\mathrm{E}_{3}}, b_{26}=V_{\mathrm{E}_{6}}, b_{20}$ $=-V_{\mathrm{E}_{0}}$, and

$$
\begin{aligned}
E_{9}+E_{3} & =E_{7} \\
E_{0}+E_{3} & =E_{8} \\
E_{3} & =K_{\text {eq }} E_{9} E_{0}
\end{aligned}
$$

There is another set of equations that relates to the fluxes, as we saw in Section 2.3 , but for simplicity we need only consider the concentration variables at this point.

Before applying these equations in an analysis of the reference system, one must first verify that the assumptions used in their derivation hold for the system being analyzed. For example, the summation relationships were derived in MCT by assuming that all enzyme levels and activities can be changed independently $[6,7,13,14]$. In the reference system of Figure 1 this is impossible to realize. A doubling of $E_{9}$ and $E_{0}$ implies a quadrupling of $E_{3}$, and Eqs. (34)-(37) are invalid.

Since the enzyme levels are not all independent of each other, one might try to introduce the dependencies, as is done in the explicit variants of BST [39]. In order to account for dependencies among the metabolite concentrations, the early MCT was generalized by the implicit procedure [5] corresponding to the explicit procedure in BST $[20,27,28]$. By a similar implicit procedure, one can relate changes in the three enzyme levels $E_{3}, E_{9}$, and $E_{0}$ to changes in the two independent variables $E_{7}$ and $E_{8}$ :

$$
\frac{\partial E_{k}}{E_{k}}=\frac{\partial E_{k}}{\partial E_{7}}\left(\frac{E_{7}}{E_{k}}\right) \frac{\partial E_{7}}{E_{7}}+\frac{\partial E_{k}}{\partial E_{8}}\left(\frac{E_{8}}{E_{k}}\right) \frac{\partial E_{8}}{E_{8}}, \quad k=3,9,0 .
$$


Thus, one obtains the relationships

$$
C_{\mathrm{E}_{k}}^{\mathbf{X}_{i}}=\frac{C_{\mathrm{E}_{7}}^{\mathbf{X}_{i}} C_{\mathrm{E}_{8}}^{\mathbf{X}_{i}}}{C_{\mathrm{E}_{7}}^{\mathbf{X}_{i}} C_{\mathrm{E}_{8}}^{\mathrm{E}_{k}}+C_{\mathrm{E}_{8}}^{\mathbf{X}_{i}} C_{\mathrm{E}_{7}}^{\mathrm{E}_{k}}}, \quad i=1,2 ; k=3,9,0,
$$

where $C_{\mathrm{E}_{7}}^{\mathrm{E}_{k}}$ and $C_{\mathrm{E}_{8}}^{\mathrm{E}_{k}}$ are new types of "control coefficients" that relate relative change of dependent enzyme levels to relative change in independent enzyme levels. These types of coefficients, treated as logarithmic gains in BST (e.g., Savageau [23]), previously have not been treated in MCT. They can be calculated directly from the constraints in Eqs. (38)-(40), e.g., by taking the relevant partial derivatives, inserting the steady-state values for the enzyme species, and solving the resulting set of linear equations.

The new relationships in Eq. (41) then can be used to rewrite Eqs. (34)-(37). For example, Fq. (34) becomes

$$
C_{\mathrm{E}_{\mathrm{o}}}^{\mathbf{X}_{i}}+\sum_{k} \frac{C_{\mathrm{E}_{7}}^{\mathbf{X}_{i}} C_{\mathrm{E}_{8}}^{\mathbf{X}_{i}}}{C_{\mathrm{E}_{7}}^{\mathbf{X}_{i}} C_{\mathrm{E}_{8}^{k}}^{\mathrm{E}_{k}}+C_{\mathrm{E}_{\mathrm{8}}}^{\mathbf{X}_{i}} C_{\mathrm{E}_{7}}^{\mathrm{E}_{k}}}=0, \quad i=1,2 ; k=3,9,0 .
$$

The use of such equations for predicting values of the "control coefficients" in the system is complicated by the nonlinear relationships that have been introduced.

Before proceeding with a detailed analysis, one should test the empirical data from the reference system against one of these equations, for instance the concentration summation relationship for $X_{1}$. Inserting the appropriate values for the new control coefficients $\left(C_{\mathrm{E}_{7}}^{\mathrm{E}_{3}}=0.449, C_{\mathrm{E}_{7}}^{\mathrm{E}_{9}}=1.68, C_{\mathrm{E}_{7}}^{\mathrm{E}_{0}}=-1.23\right.$, $\left.C_{\mathrm{E}_{8}}^{\mathrm{E}_{3}}=0.754, C_{\mathrm{E}_{8}}^{\mathrm{E}_{9}}=-0.922, C_{\mathrm{E}_{8}}^{\mathrm{E}_{0}}=1.68\right)$ and taking the empirical values of $C_{\mathrm{E}_{6}}^{\mathrm{X}_{1}}, C_{\mathrm{E}_{7}}^{\mathrm{X}_{1}}$, and $C_{\mathrm{E}_{8}}^{\mathrm{X}_{1}}\left(L_{16}, L_{17}\right.$, and $\left.L_{18}\right)$ from Table 1, one finds for Eq. (42) that

$$
-0.768-0.719+0.303-0.213=-1.40 \neq 0
$$

This type of test shows that MCT cannot be generalized by introducing the dependencies. The reason is that the original "summation theorems," which were the starting point in this development, are invalid, even if the newly defined "control coefficients" are valid.

Another approach to generalization would be to abandon the original "summation theorems" and ask if one can find some other summation among the control coefficients involving only changes in independent enzyme levels. In the simplest case,

$$
C_{\mathrm{E}_{6}}^{\mathbf{X}_{i}}+C_{\mathrm{E}_{7}}^{\mathbf{X}_{i}}+C_{\mathrm{E}_{8}}^{\mathbf{X}_{i}}=0, \quad i=1,2 .
$$

The answer is again no, as can be seen by inserting the empirical values 
taken from Table 1. The reason is that, although the enzyme levels $E_{6}, E_{7}$, and $E_{8}$ are now independent variables, the reactions are not linear in these independent variables.

There seems to be no straightforward way to modify the MCT approach by generalizations of the type that have been used in the past. The fundamental "theorems" are invalid for biochemical systems in which reaction rates are not linear in enzyme levels and the enzyme levels are not independent. If one wants to pursue the development of a general implicit representation that would be valid for realistic systems, then one must start from first principles and derive a different set of relationships, without making the restrictive assumptions found in MCT.

The Implicit GMA Variant. As is always the case within BST, one starts the development with the equations for the conservation of mass and the constraints on system constituents, in this case the total enzyme concentrations [Eqs. (3)-(7)]. However, in the implicit variants the rate laws and constraints are not written explicitly as power-law functions, and so the analysis proceeds by implicit differentiation of these functions.

As with the explicit variants within BST (see Part I [39] and Section 2), the development of the implicit variants can follow two different routes depending on the level of aggregation used to represent the different processes. Aggregation at the level of individual enzyme-catalyzed reactions will produce an implicit GMA representation, while aggregation at the level of net synthesis/net degradation for each system constituent will produce an implicit S-system representation. Since aggregation at the level of individual enzyme-catalyzed reactions is assumed in MCT, the appropriate generalization is the implicit GMA variant within BST (see Section 4 for additional commentary on the implicit S-system variant).

To understand the relation between MCT and its generalization by means of the implicit GMA variant, it is of value to contrast the enumeration of equations in the implicit GMA variant [Eqs. (3)-(7)] with that in MCT. Since the implicit GMA variant is closely related to the explicit GMA variant, the implicit representation also involves $n$ equations in $n$ unknowns (see Section 2). On the other hand, any solution in MCT involves the fundamental summation and connectivity relationships, which implies $r$ equations in $r$ unknowns (not the $r(r+b)$ stated by Crabtree and Newsholme [4], because one does not have to solve the control coefficients for $r+b$ system variables simultaneously; e.g., see [5]). In any case, the size of the system that must be solved simultaneously is larger than the minimum $n$ determined by standard criteria from network topology. The difference is $r=4$ versus $n=2$ in the case of the reference system in Figure 1 with the enzyme association/dissociation at steady state, which is the case considered in MCT. 
Just as the explicit GMA variant within BST provides the most efficient representation for the class of theories that includes FOT, so the implicit GMA variant within BST provides the most efficient representation for the class of theories that includes MCT.

\subsection{BEHAVIOR OF THE CONCENTRATIONS IN STEADY STATE}

As in the case of the explicit variants, the starting point for this analysis is the steady-state equations corresponding to Eqs. (3) -(7).

$$
\begin{array}{r}
v_{41}-v_{12}=0 \\
v_{12}+v_{42}-v_{25}=0 \\
v_{03}-v_{30}=0 \\
X_{9}=X_{7}-X_{3} \\
X_{0}=X_{8}-X_{3}
\end{array}
$$

One cannot obtain an analytical solution for the $X_{i}$ in these equations, which eliminates the possibility of a direct method for characterizing the steady-state behavior. To characterize the response of the system in the absence of explicit functional forms for the rate laws, one must proceed by implicit differentiation of the steady-state equations.

Logarithmic Gains. To characterize the steady-state response to change in an independent variable (say $X_{6}$ ), one takes the logarithmic derivative of the steady-state equations with respect to the independent variable, i.e., one differentiates the steady-state equations with respect to the independent variable, multiplies through by the independent variable and divides through by the value of the function itself, and evaluates the expressions at the steady-state operating point. Following this procedure, one obtains, for the last two constraint equations [Eqs. (46) and (47)]

$$
\frac{\partial X_{9}}{\partial X_{6}}\left(\frac{X_{6}}{X_{9}}\right)=\frac{\partial X_{9}}{\partial X_{3}}\left(\frac{X_{3}}{X_{9}}\right) \frac{\partial X_{3}}{\partial X_{6}}\left(\frac{X_{6}}{X_{3}}\right),
$$

and

$$
\frac{\partial X_{0}}{\partial X_{6}}\left(\frac{X_{6}}{X_{0}}\right)=\frac{\partial X_{0}}{\partial X_{3}}\left(\frac{X_{3}}{X_{0}}\right) \frac{\partial X_{3}}{\partial X_{6}}\left(\frac{X_{6}}{X_{3}}\right) .
$$

By the conventions in the power-law formalism (see Part I [39] and Section 2 ), these equations can be recognized as

$$
L_{96}=f_{93} L_{36} \quad \text { and } \quad L_{06}=f_{03} L_{36} \text {, }
$$


where by definition $L$ is the relative change in a dependent concentration variable with respect to the relative change in an independent variable, and $f$ is the relative change in one constraint variable with respect to the relative change in another [see Eqs. (18) and (19) in Part I].

Similarly, one obtains for the first equation [Eq. (43)] ${ }^{6}$

$$
\begin{gathered}
\frac{\partial v_{41}}{\partial X_{9}}\left(\frac{X_{9}}{v_{41}}\right) \frac{\partial X_{9}}{\partial X_{6}}\left(\frac{X_{6}}{X_{9}}\right)+\frac{\partial v_{41}}{\partial X_{1}}\left(\frac{X_{1}}{v_{41}}\right) \frac{\partial X_{1}}{\partial X_{6}}\left(\frac{X_{6}}{X_{1}}\right)-\frac{\partial v_{12}}{\partial X_{1}}\left(\frac{X_{1}}{v_{12}}\right) \frac{\partial X_{1}}{\partial X_{6}}\left(\frac{X_{6}}{X_{1}}\right) \\
-\frac{\partial v_{12}}{\partial X_{2}}\left(\frac{X_{2}}{v_{12}}\right) \frac{\partial X_{2}}{\partial X_{6}}\left(\frac{X_{6}}{X_{2}}\right)-\frac{\partial v_{12}}{\partial X_{0}}\left(\frac{X_{0}}{v_{12}}\right) \frac{\partial X_{0}}{\partial X_{6}}\left(\frac{X_{6}}{X_{0}}\right)=0
\end{gathered}
$$

If one inserts Eqs. (48) and (49) into this expression and again uses the conventions in the power-law formalism, then it can be recognized as

$$
g_{19} f_{93} L_{36}+g_{11} L_{16}-g_{211} L_{16}-g_{221} L_{26}-g_{201} f_{03} L_{36}=0
$$

or

$$
\left(g_{11}-g_{211}\right) L_{16}-g_{221} L_{26}+\left(g_{13}-g_{231}\right) L_{36}=0
$$

since $g_{13}=g_{19} f_{93}$ and $g_{231}=g_{201} f_{03}$ [see Eqs. (13)-(15)]. The remaining equations [Eqs. (44) and (45)] in the set follow in a similar fashion:

$$
g_{211} \frac{v_{12}}{v_{25}} L_{16}+\left(g_{221} \frac{v_{12}}{v_{25}}+g_{222} \frac{v_{42}}{v_{25}}-h_{22}\right) L_{26}+\left(g_{231} \frac{v_{12}}{v_{25}}+g_{232} \frac{v_{42}}{v_{25}}\right) L_{36}=h_{26}
$$

$$
a_{33} L_{36}=0
$$

Although no explicit use of the power-law formalism has been made, the above procedure leads to a set of equations that are identical to Eqs. (19)-(21), which were obtained by the explicit method. This equivalence follows from the origins of the power-law formalism and the meaning of kinetic order as the ratio of differential change in a logarithmic coordinate system.

\footnotetext{
${ }^{6}$ In the indirect approach one is rederiving the power-law formalism implicitly in the process and one must be careful to distinguish the first partial derivative in each term from the second. The first involves differentiation with respect to one variable while all other variables are held constant; in the second, one differentiates with respect to one independent variable while all other independent variables are held constant.
} 
Parameter Sensitivities. In the implicit GMA variant, one cannot develop a set of equations similar to Eqs. (22)-(24) and (25)-(27) for the parameter sensitivities. For this one must know what the parameters are and how they enter into the rate laws in order to calculate the required derivatives. This means knowledge of the underlying formalism, which is not available unless the power-law formalism is made explicit. This loss of information limits the characterization of the steady state that one can obtain by using implicit variants.

\subsection{BEHAVIOR OF THE FLUXES IN STEADY STATE}

For any given flux, one can differentiate the expression with respect to an independent concentration variable and implicitly determine the relationships between logarithmic gains for fluxes and those for concentrations.

$$
\begin{aligned}
L\left(V_{i r}, X_{k}\right) & =\frac{\partial V_{i r}}{\partial X_{k}}\left(\frac{X_{k}}{V_{i r}}\right)+\sum_{j=1}^{3} \frac{\partial V_{i r}}{\partial X_{j}}\left(\frac{X_{j}}{V_{i r}}\right) \frac{\partial X_{j}}{\partial X_{k}}\left(\frac{X_{k}}{X_{j}}\right) \\
& =g_{i k r}+\sum_{j=1}^{3} g_{i j r} L\left(X_{j}, X_{k}\right), \quad i=1,2,3 ; k=4, \ldots, 8 ; r=1,2 .
\end{aligned}
$$

Thus, once the concentration behavior has been determined, the flux behavior, at least in response to changes in the independent concentrations, follows directly. As in the case of concentrations, one cannot obtain the parameter sensitivities in the implicit variants.

\subsection{BEHAVIOR ABOUT THE NOMINAL STEADY STATE}

The summation and connectivity relationships, which are the primary equations in MCT, contain only one of the two fundamental types of parameters, kinetic orders but not rate constants, in the underlying power-law formalism. Hence, the mathematical representation used in MCT allows neither an explicit symbolic solution nor a numerical solution of the global behavior in terms of the underlying parameters and independent variables. This is also true for the more general equations of the implicit GMA variant within BST, in contrast to the situation with the explicit variants. Sorribas and Bartrons [38], however, have shown that one can assume a steady state, derive a linear representation of the system, and relate its stability properties to the kinetic orders and steady-state fluxes of the system expressed in the nomenclature of MCT. One starts with the differential equations that correspond to the dynamics of the system [Eqs. (3)-(5)], instead of using the 
fundamental theorems of MCT. The dynamic equations can be written

$$
\frac{d X_{i}}{d t}=\sum_{k} \rho_{i k} v_{k}
$$

where $\rho_{i k}=1$ if $v_{k}$ produces $\mathrm{X}_{i}, \rho_{i k}=-1$ if $v_{k}$ consumes $\mathrm{X}_{i}$, and $\rho_{i k}=0$ otherwise. (For simplicity we are assuming that the stoichiometric values are unity; if this is not the casc, then one replaces the integer 1 by the appropriate stoichiometric values.) Although this representation has no fundamental advantage over that in Eqs. (3)-(5), it is useful for expressing results in the nomenclature of MCT. By using standard procedures, one can write the linearized version of the above equation as

$$
\frac{d u_{i}}{d t}=\sum_{j=1}^{n}\left[\sum_{k} \rho_{i k} \epsilon_{\mathbf{X}_{j}} \frac{v_{k 0}}{X_{i 0}}\right] u_{j},
$$

where $u_{i}=\left(X_{i}-X_{i 0}\right) / X_{i 0}, \epsilon_{\mathrm{X}_{i}}^{\mathrm{E}_{k}}$ represents the elasticity of $v_{k}$ with respect to $\mathrm{X}_{j}$, and the additional zero subscript signifies steady-state values.

As in the case of the explicit GMA variant, this equation allows one to discuss the necessary conditions for local stability by applying the Routh criteria. When one takes into account the identical meaning of the kinetic orders in all three variants, one sees that

$$
\frac{\sum_{k} \rho_{i k} \epsilon_{\mathrm{X}_{j}}^{\mathrm{E}_{k}} v_{k 0}}{\sum_{p} v_{p 0}}=\sum_{r} g_{i j r} \frac{V_{i r 0}}{V_{i 0}}-\sum_{r} h_{i j r} \frac{V_{-i r 0}}{V_{i 0}}=a_{i j},
$$

where the left-hand side refers to the implicit GMA variant (expressed in the notation of MCT), the sum over $k$ includes all reactions in the system and the sum over $p$ includes all reactions that contribute to the synthesis of $\mathrm{X}_{i}$, the middle terms refer to the explicit GMA variant and the sum over $r$ includes all reactions that contribute to the synthesis and degradation of $\mathbf{X}_{i}$, and the right-hand side refers to the S-system variant and $a_{i j}=g_{i j}-h_{i j}$. Thus, Eq. (54) can be written as

$$
\frac{d u_{i}}{d t}=\sum_{j=1}^{n} \frac{V_{i 0}}{X_{i 0}} a_{i j} u_{j},
$$

which is identical to that obtained for the explicit S-system variant within 
BST [26], and thus the conditions for local stability also are identical to those for the S-system variant $[26,27]$.

\subsection{RESULTS}

Logarithmic Gains. The responses of the dependent variables $X_{1}, X_{2}$, $X_{3}, V_{1}, V_{2}$, and $V_{3}$ to changes in the independent variables $X_{4}, X_{5}, X_{6}, X_{7}$, and $X_{8}$, are obtained by solving sets of equations such as Eqs. (50)-(53). The results are identical to those obtained previously with the explicit S-system and generalized mass action variants (see Table 1). It is important to point out that the analysis of the reference system using the implicit GMA variant has been performed without reference to the "control theorems" of MCT. In fact, relationships with the meaning of these "theorems" in MCT cannot be defined for systems that include enzyme-enzyme interactions, because such mechanisms generate nonindependent rate laws that are nonlinear functions of enzyme concentration or activity. The general set of constraint relationships that are valid in the power-law formalism already are implicit in the steady-state solution $[33,37]$. These relationships are a property of the mathematical representation, rather than a property uniquely associated with the biochemical systems being studied.

Parameter Sensitivities. As indicated in Sections 3.2 and 3.3, there are no results with the implicit approach that correspond to those given in Tables 2 and 3 for the steady state; these types for results can be obtained only with the explicit approaches.

Behavior about the Nominal Steady State. There also are no results for the local behavior in the neighborhood of the steady state that are comparable to those given in Table 4 and Figures 2 and 3, which again were obtained with the explicit approaches.

Summary of Results. The results in this section show that the summation and connectivity theorems, the fundamental principles that characterize MCT, are invalid for biochemical systems that include enzyme-enzyme interactions. These theorems have no inherent relation to the biochemical system; rather, they reflect a particular mathematical description that does not hold for the reference system in Figure 1 or for biochemical systems in general. There is no possibility of making minor adjustments in this theory; a radical change is required that involves rejection of its fundamental principles-the summation and connectivity theorems. As shown above, an appropriate generalization that does not involve the fundamental principles of MCT or its limiting assumptions is provided by the implicit GMA variant within BST.

The results obtained by analysis using the implicit GMA variant are included as a subset of those obtained by analysis using the explicit GMA 
variant within BST. That is, one obtains the same results in situations where comparable analyses can be carried out [compare Eqs. (19)-(21) and (28) with (50)-(52) and (53)]. The principal operational difference is that in the implicit variant one is rederiving the power-law formalism each time one carries out an analysis. ${ }^{7}$ In the explicit use of the power-law formalism, the derivation of the formalism has been given once and for all. One writes the equations in the power-law formalism by inspection of the mechanism and, when necessary, substitutes the appropriate constraint expressions.

There are other differences as well. For example, there are no expressions in the implicit variant that correspond to Eqs. (22)-(24), (29), and (30) in the explicit variant. This is because there are no rate-constant parameters identified in the implicit variant. Similarly, there are no expressions in the implicit variant that correspond to Eqs. (25)-(27), (31), and (32). In this case one has difficulty carrying out the required operations without knowing the functional form, as is the case in the implicit variant. Only in the explicit variants can one solve the equations numerically to obtain the system behavior in a local region about the nominal steady state. This is because one has a full representation in terms of all the component parameters, including the rate constants as well as the kinetic orders.

\section{DISCUSSION}

We have analyzed the reference system in Figure 1 using three variant theories based upon the same underlying power-law formalism: (1) the explicit S-system variant presented in Part I [39], (2) the explicit GMA variant, which includes FOT as a special case, and (3) the implicit GMA variant, which is seen to be a subset of the second approach and also to include MCT as a special case. All these theories may be considered variants of a single theory, BST. The logical relationships among the variant theories are summarized in Figure 4. BST is based on the power-law formalism and can be manifested in two forms. In one the kinetic descriptions of the underlying components are explicit, while in the other these descriptions are implicit. In all cases, the first step in applying BST is

(1) Formulation of the basic network equations, which involve Kirchhoff's flux laws, rate laws for the component processes, and constraints among the constituents of the system.

\footnotetext{
${ }^{7}$ This is true for all implicit variants. For example, Kacser [12] rederives the power-law representation for the component reactions in his example analyzing a simple branch point. It should be noted that this procedure is identical to that presented by Heinrich and Rapoport [7] and gives results that are equivalent to those in FOT (compare Eq. (4) in Kacser [12] with the equation at the middle of page 20 in Crabtree and Newsholme [2]). The general significance of this procedure has not been recognized in MCT, which continues to treat the relationships derived by this procedure as auxiliary while maintaining the centrality of the "control theorems" (e.g., see [15]).
} 


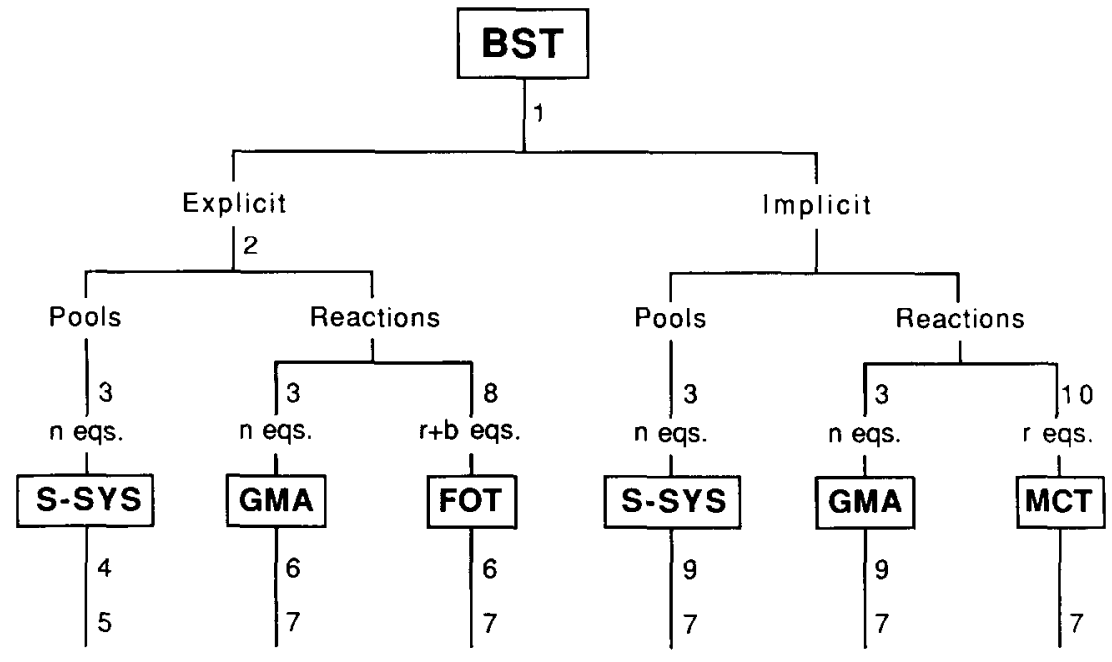

FIG. 4. Logical relationships among the variants within biochemical systems theory (BST). The first major distinction among variants is whether the underlying power-law formalism is explicit or implicit. The second is whether aggregation is at the level of net flux through each constituent pool or at the level of net flux through each enzyme-catalyzed reaction. The third is whether the mathematical equations that characterize the system are combined in a fully reduced form to give the minimum number known from standard network theory [S-system (S-SYS) variant, generalized mass action (GMA) variant] or in a partially reduced form that gives a larger number than necessary [flux-oriented theory (FOT) variant, metabolic control theory (MCT) variant]. The number of dependent metabolities is $n$, which is also the minimum number of equations necessary to characterize the system. The number of reactions is $r$, and the number of branch points is $b$, where $n<r<r+b$. The numbers 1-10 refer to the principal steps in the application of BST that are outlined in Section 4.

The next step in the explicit forms of the theory is

(2) Explicit representation of the component processes and constraints in the power-law formalism under in situ conditions.

Then, depending on how one chooses to aggregate the fluxes in the system, onc obtains a number of different representations $[20,27,46]$. The two most important representations used for biochemical systems involve aggregation at the level of net flux through each constituent pool of the system or aggregation at the level of net flux through each enzyme-catalyzed reaction in the system.

Steady-state analysis using the explicit S-system variant, which involves aggregation of flux through each pool, proceeds by the following steps.

(3) Expression of the system equations in the optimal or fully reduced form that is known from network theory to minimize the effort of solution, 
(4) Explicit solution of the steady-state equations in the power-law formalism, and

(5) Partial differentiation of the solution with respect to independent concentrations and system parameters in order to highlight particular systemic responses.

This five-step procedure exhibits many advantages of the explicit S-system variant.

Step 3 gives the explicit GMA variant when aggregation is at the level of net flux through each enzyme-catalyzed reaction. In this explicit variant of BST, one cannot obtain an explicit steady-state solution, and so the steadystate analysis proceeds by the following steps:

(6) Partial differentiation with respect to independent concentrations and system parameters of the explicit steady-state equations in the power-law formalism.

(7) Solution of the resulting linear algebraic equations for the logarithmic gains and parameter sensitivities of interest in characterizing systemic responses.

One loses some advantages of the explicit S-system variant by this procedure; nevertheless, one can obtain fairly complete numerical results for specific cases.

The FOT variant is generated by the following step:

(8) Nonoptimal or partial reduction of equations and introduction of restrictive assumptions produce a special case of the explicit GMA variant.

Thus, subsequent steps in the analysis follow the same sequence indicated above for the explicit GMA variant (steps 6 and 7).

In the implicit forms of the theory, one deals only with steady-state issues. In most other respects the development is logically similar to that of the explicit GMA variant, although steps 2 and 6 are not clearly separated as they are in the explicit GMA variant. Instead, one aggregates according to fluxes through pools (S-system variant) or fluxes through enzyme-catalyzed reactions (GMA variant), expresses the equations in the optimal fully rcduced form (step 3), and then carries out step 9:

(9) Differentiation of the steady-state equations in their implicit form, which involves a mixture of types of partial differentiation, and simultaneous derivation of the power-law representation for the component processes and generation of a set of linear equations in either logarithmic gains or parameter sensitivities.

Solution of the resulting equations is then accomplished in the conventional manner (step 7). 
The MCT variant is generated by step 10:

(10) Use of restrictive assumptions leading to summation and connectivity relationships that correspond to special cases of the orthogonality properties inherent within the GMA variant. These relationships yield a larger set of equations than the minimum necessary for efficient solution.

Thus, the MCT variant is a special case of the implicit GMA variant, and the solution of the resulting equations is accomplished in the conventional manner (step 7).

Although the resulting implicit variants are logically related to the corresponding explicit variants, they are operationally distinct. Because information contained in the rate constants is lost and one does not have an explicit representation of the underlying kinetic equations in the implicit variants, the results that can be obtained by these variants represent a restricted subset of those available with the explicit variants.

In summary, the logical framework within BST (Figure 4) provides a context for rigorous comparisons of variant theories that are based upon the underlying power-law formalism.

Integrated biochemical systems are inherently complex. Hence it is desirable, indeed inevitable, that simplifications be introduced for their study. This also implies that compromises must be made and that these must be evaluated to ensure that the simplifications introduced are judicious ones. Each of the approaches considered in these papers involves such compromises, and thus each has its advantages and disadvantages.

\subsection{METABOLIC CONTROL THEORY}

Metabolic control theory (MCT) was introduced four or five years after the introduction of BST and is based on the same power-law formalism [36]. MCT was the first of these approaches to emphasize constraint relationships, which are now known to be the familiar orthogonality properties of linear systems, and show that these provide another perspective on the interrelatedness of component processes in intact biochemical systems [37]. This perspective has made available another route for characterizing the distribution of influences in a complex system of biochemical reactions [14]. This approach has focused on certain simple classes of biochemical systems, and thus the analysis in these cases is correspondingly simple and attractive. In this way MCT probably has helped to popularize the study of integrated biochemical systems.

In several respects MCT appears to be different from other approaches, and so their relatedness generally has not been recognized [42]. This undoubtedly is the result of several factors, the most prominent being the implicit rather than explicit use of the power-law formalism and the nearly exclusive focus on specific constraint relationships called control theorems. 
These factors have made it more difficult to evaluate the inherent strengths and limitations of MCT vis-à-vis other approaches and have led to a certain amount of confusion in the field.

First, there has been confusion concerning the implications of implicit versus explicit use of the power-law formalism. The implicit approach of MCT has been interpreted $[8,14]$ as being more general than the approach of BST, which involves the power-law representation explicitly. Making the kinetic description explicit appears to involve a restrictive assumption, while leaving the kinetic description implicit suggests that one is not making any assumptions about the underlying kinetic description. The results in Section 3 demonstrate just the reverse. Leaving the representation implicit does not yield greater generality; it reduces generality and restricts application to a single steady-state point. Furthermore, one cannot completely characterize the system's behavior at this point. For example, one cannot examine how the steady-state behavior will change as a consequence of variation in the system's parameter values (Tables 2 and 3). One also cannot predict the steady-state (Figure 2) or dynamic (Figure 3) behavior of a system in a local neighborhood about the nominal steady state. These conclusions are true not only for MCT, but for all implicit approaches, including the more general implicit S-system and implicit GMA variants within BST.

Second, there has been some confusion as to the appropriate role of the summation and connectivity theorems. In the absence of an explicit representation for the underlying kinetics, MCT has focused on a special mathematical representation based on constraint relationships called control theorems, which are a special case of the orthogonality properties of linear systems $[33,37]$. These theorems were derived from a set of specific definitions and simplifying assumptions. The most critical were the assumption that enzyme concentrations and molecular activities can be considered independent variables identified with a single associated reaction in the system, and the assumption that the individual reactions in the system are linear in the enzyme concentrations and molecular activities [13, 14]. Despite these restrictive assumptions, the "control theorems" are considered incorrectly to be generally applicable: "Rigorous and logical analysis of the behaviour of metabolic systems demonstrated that this summation equals unity, whatever the complexity of the system.... The sum of all concentration control coefficients for any one metabolite is always zero, no matter how complex the metabolic system may be" [15].

To cover new aspects not considered in earlier versions, MCT has been extended over the years. In the process, the simplicity of the original summation and connectivity relationships has been lost as new parameters and interpretations have had to be introduced (e.g., see [8], [18]). Nevertheless, the summation and connectivity relationships have remained fundamental to the MCT approach throughout its development $[5-8,13-15,18,47$, 
48]. Indeed they are considered to be essential for understanding biochemical systems: "The various summation and connectivity properties... are... fundamental to any discussion of metabolic control.... Discussion and experiment in the absence of an understanding of these summation and connectivity properties can only proceed in a kind of intellectual vacuum" [15]. Similarly, Westerhoff and Kell [48] have emphasized these theorems as the "principles of Metabolic Control Theory." This nearly exclusive focus on the control theorems in MCT is, however, self-limiting. The results in this paper show that the control theorems of MCT are not valid for at least one of the more important classes of biochemical systems (see also [33], [37]). Hence, MCT is not appropriate as a foundation on which to build a theory of biochemical systems.

In order to provide the most favorable evaluation of its scope and the clearest demonstration of its relatedness to other approaches, we have had to generalize MCT. Minor changes that preserve a role for the fundamental theorems of MCT were shown to be inadequate for biochemical systems involving enzyme-enzyme interactions. A more appropriate generalization was provided by the implicit GMA variant within BST, which was shown to include MCT as a special case. This generalization of MCT removes a number of restrictive assumptions and makes the resulting theory applicable to the same class of biochemical systems as BST. Nevertheless, it remains an implicit approach, and, as indicated above, this limits one's ability to characterize the integrated behavior of the biochemical system.

\subsection{FLUX-ORIENTED THEORY}

The flux-oriented theory of Crabtree and Newsholme was introduced four or five years after the introduction of MCT and is based more directly on the power-law formalism. This approach was the first to emphasize explicit power-law kinetics in a GMA-like representation. The fact that it is an explicit approach accounts for most of its advantages. Nevertheless, the relatedness of FOT to MCT and BST generally has not been appreciated. In part this is because FOT is the most recently developed of these approaches, and its relative strengths and weaknesses are only now being determined through appropriate comparisons with the other theories.

The fact that MCT is an implicit approach perhaps makes it more difficult to recognize that FOT and MCT are closely related. The areas of overlap between implicit approaches, of which MCT is a special case, and explicit approaches, of which FOT is a special case, can be seen by comparing the results of specific application to the reference system in Figure 1. Both approaches yield exactly the same predictions for logarithmic gains (Table 1). In this area, even the more general implicit GMA variant within BST produces nothing fundamentally new that is not provided by the explicit variants within BST. 
This is not to say that the FOT and MCT approaches are identical. Making the power-law formalism explicit allows one to perform a deeper analysis and make additional predictions not possible when the power-law formalism is only implicit (see comments under Section 4.1). For example, the explicit approach is capable of making predictions about the parameter sensitivities of the system (Tables 2 and 3 ) and predicting the local behavior of the system in the neighborhood of the nominal steady state (Figures 2 and 3 ), although these types of predictions have not been made in FOT. Thus, from the results in this paper one can conclude that MCT is more limited than FOT. By using MCT one obtains only a subset of the results that can be obtained by using FOT. This central fact has not been noted in previous comparisons of these two approaches [Trends Biochem. Sci. 12:5-14, 216-224 (1987)].

The relatedness of FOT and BST may have been overlooked because of differences in representation within the power-law formalism. FOT is based on an explicit representation in which flux is considered in terms of each enzyme-catalyzed reaction, whereas in BST the emphasis has been on the S-system representation in which flux is considered in terms of each metabolic pool. Nevertheless, the two approaches are very similar. The approach that Crabtree and Newsholme [2-4] have described draws heavily on power-law functions, and many of their theoretical developments have confirmed those presented in BST. For example, in cases where there are dependencies among the metabolic concentrations due to functional algebraic constraints (e.g., among ATP, ADP, and AMP when the total adenylate pool is constant), one must include these in an appropriate fashion within the system's equations. Their method of representing such a sum in the power-law formalism [3] is identical to that given by Savageau [27 (Chap. 5), 28].

The parallelism runs much deeper. If one avoids restrictive assumptions concerning the linearity and independence of enzyme activities, saturation of specific reactions, operation near equilibrium, etc. (while these may apply in specific cases, they are not generally valid), and if one adopts the nomenclature that is conventionally used in network theories, then one can see that FOT readily generalizes to the explicit GMA variant within BST. The fundamental difference between the explicit GMA variant and the explicit S-system variant within BST is the following: steady-state behavior in the GMA variant is governed by nonlinear relationships that cannot be simplified, whereas in the S-system variant it is governed by nonlinear relationships that can be readily transformed into simpler linear relationships in the logarithms of the variables. This fundamental difference has a host of implications. Most of these have been discussed at length elsewhere (see Voit and Savageau [46] and Savageau et al. [36,37]), so we shall review only the most important. 
In the GMA variant one cannot make well-controlled comparisons involving explicit symbolic constraints on global behaviors. By contrast, one can easily generate such constraints in the S-system variant by simply equating the explicit steady-state solution that corresponds to the global property in question for each system. In this way one can draw very general conclusions, independent of specific parameter values, from comparisons that correspond to a "well-controlled" experiment (e.g., see Savageau [24, 25, 27] and Savageau and Jacknow [32]). Such well-controlled comparisons have been used in BST to deduce very general conclusions regarding the evolution of alternative designs for regulatory mechanisms in genetic circuits [27] and immune networks $[9,10]$, as well as biochemical pathways (for a brief review see Savageau [30]).

Even if one focuses only on specific numerical cases, as opposed to general class-specific properties, the GMA variant has limitations when compared to the S-system variant. In the GMA variant there is no proof that a steady-state solution can be obtained by numerical methods $[16,17,49]$. Furthermore, numerical solutions, when they exist, are not obtained as efficiently as they are in the S-system variant, which is supported by the specific calculations required for the comparisons in Figures 2 and 3 (data not shown). For a more general analysis of this subject see [11] and Irvine and Savageau [in preparation].

Finally, although the range of accurate representation by the GMA variant has been demonstrated to be considerably greater than the $10 \%$ limit mentioned by Crabtree and Newsholme [2], this range is less than that by the S-system variant (e.g., see Voit and Savageau [46] and the results in Figure 2 and Table 4 for the specific reference system in Figure 1).

\subsection{BIOCHEMICAL SYSTEMS THEORY}

Biochemical systems theory (BST) has been compared to older approaches involving the linear formalism and the Michaelis-Menten formalism [24, 27]. The theoretical development of BST and its application to a variety of biological systems have been reviewed (e.g., see Savageau [24, 27, $29,30]$ and Savageau and Voit [34]), and the more important advances have been summarized in Part I [39]. In this section we shall comment on three limitations of BST and on new opportunities for development within the power-law formalism that address these limitations.

The first and most characteristic limitation of BST, like that of all local representations, is the range of variation in concentrations over which the representation is valid. Although this range is far broader for the S-system variant within BST than for other well-known local representations, and although it is comparable to the ranges that are exhibited experimentally in biochemical systems, it nonetheless is a potential problem for some systems. 
A related issue that is evident from the comparisons in this paper is the monotonic nature of the responses in steady state. One can accurately represent the behavior of a system with nonmonotonic behavior for small variations about a steady state on the rising portion or the falling portion of its characteristic, but the representation cannot be expected to cover a range of variation that encompasses both the rising and falling phases of a response.

What are the alternatives when one encounters situations such as these? One obvious choice is to fall back on a more complex nonlinear strategy, such as representation by rational functions. This will provide acceptable numerical descriptions for specific systems, but its limitations for revealing more general class-specific properties of biochemical systems are well known (e.g., see Savageau [24, 27]). Another choice, for which there is ample precedent, is "piecewise representation." One can subdivide the actual range of operation into smaller ranges within which the behavior is monotonic and accurately represented by separate power-law functions. This is a straightforward extension of the well-known methods for piecewise linear representation [1b]. Again, this will lead to acceptable numerical descriptions for specific systems, but by introducing an ad hoc subdivision into the theory one loses its coherent and general characteristics. An alternative to these ad hoc approaches has recently become available as a result of developments within the power-law formalism itself $[28,35,43,45]$. It is now possible to remain within the power-law formalism and, by introducing additional variables, improve the range of representation or in fact achieve an exact representation for the nonlinear functions likely to be encountered in any biochemical system. This approach has the advantage of staying within a unified theoretical framework. It also means that many of the powerful methods already developed for efficient solution of the equations in the power-law formalism can be applied here as well.

The second major limitation, following these problems of accurate representation, is in the methods for solving the full dynamic problem in BST. Unlike the steady-state solution, which is straightforward because it represents a linear problem when transformed into logarithmic coordinates, the dynamic solution involves nonlinear differential equations. This is a fundamental problem for any rcalistic formalism. In general, one cannot obtain such solutions except for specific numerical cases, and even for these the available methods are often unsatisfactory in terms of reliability, accuracy, and efficiency. Recent developments within the powcr-law formalism have led to significant advances in the solution of this problem as well [11; Irvine and Savageau, in preparation]. As a result one can now obtain dynamic solutions with far greater reliability and accuracy one to two orders of magnitude faster than possible with other methods (see also [35]). 
The third limitation common in this field, and applicable to BST as well, is the difficulty in estimating values of component parameters from measurements on the intact biochemical system. A systematic approach to this problem has been developed recently within BST [Sorribas and Savageau, in preparation] and was used to generate (in reconstruction experiments) the component parameter values used in these papers. Although this approach is not a complete solution to the parameter estimation problem, it represents an advance over existing methods and points to new avenues for future exploration.

\subsection{CONCLUSIONS}

The evidence presented in these two papers, which results from theoretical considerations as well as from the analysis of a specific system, indicates that the explicit S-system variant provides a systematically structured formalism that brings clarity, simplicity, and directness to the analysis of complex biochemical systems. The approaches called FOT and MCT are particular cases of the GMA variant within BST. The hierarchy of inclusiveness and utility is as follows:

$$
\begin{aligned}
\text { explicit } \text { S-system } & >\text { explicil GMA }>\text { FOT }>\text { implicit S-system } \\
& =\text { implicit GMA }>\text { MCT }
\end{aligned}
$$

Neither FOT nor MCT, even in their generalized versions represented by the explicit and implicit GMA variants, provides the range of results that is provided by the explicit S-system variant within BST. Hence, the explicit S-system variant within BST represents the most general framework among the several possibilities that can be derived by the use, implicitly or explicitly, of the power-law formalism. Finally, the recent development of entitely new methodologies for the study of complex nonlinear biochemical systems demonstrates the continued fertility of biochemical systems theory and the underlying power-law formalism on which it is based.

We thank Drs. D. H. Irvine and E. O. Voit for constructive discussion and critical examination of the manuscript, and two anonymous reviewers for suggesting improvements in the presentation. This work was supported in part by grant 135-PIS from the Presidential Initiatives Fund of the University of Michigan, U.S. Public Health Service grant GM-30054 from the National Institutes of Health, and grant EET-8712756 from the National Science Foundation.

Note added in proof: Upon completion of this work we learned that Cascante, Franco, and Canela (elsewhere in this issue) have independently 
developed essentially the same generalization of the MCT approach as that involving the implicit GMA representation in this paper. Because these authors emphasize the role of sensitivity theory in their work, it might help to understand better the relatedness of their work to our own if we briefly recount the influence of sensitivity theory on the development of BST. Bode is generally considered the father of sensitivity theory, which is a part of the network theory he also was instrumental in advancing [1a]. Network theory later became a part of systems theory [1]. BST was formed in this tradition, and the terminology of gains and sensitivities [23] comes from this source. The textbook by Savageau [27] comments specifically on BST's relationship to sensitivity theory and gives reference to the collection of key papers in the development of sensitivity theory [4a]. Additional references to the relatedness of BST to sensitivity theory can be found in a recent review [30] and in recent papers comparing BST and MCT [36, 37].

\section{REFERENCES}

1 L. von Bertalanffy, General Systems Theory, George Braziller, New York, 1968.

1 a H. W. Bode, Network Analysis and Feedback Amplifier Design, Van Nostrand, Princeton, N.J., 1945

1b L. O. Chua, Introduction to Nonlinear Network Theory, McGraw-Hill, New York, 1969.

2 B. Crabtree and E. A. Newsholme, Sensitivity of a near-equilibrium reaction in a metabolic pathway to changes in substrate concentration, Eur. J. Biochem. 89:19-22 (1978).

3 B. Crabtree and E. A. Newsholme, A quantitative approach to metabolic control, Current Topics Cell. Reg. 25:21-76 (1985).

4 B. Crabtree and E. A. Newsholme, The derivation and interpretation of control coefficients, Biochem. J. 247:113-120 (1987).

4a J. B. Cruz, Ed., System Sensitinity Analysis, Dowden, Hutchinson and Ross, Stroudsburg, Pa., 1973.

5 D. A. Fell and H. M. Sauro, Metabolic control and its analysis. Additional relationships between elasticities and control coefficients, Eur. J. Biochem. 148:555-561 (1985).

6 R. Heinrich and T. Rapoport, A linear steady-state treatment of enzymatic chains, Eur. J. Biochem. 42:89-95 (1974).

7 R. Heinrich and T. A. Rapoport, Mathematical analysis of multienzyme systems 2. Steady-state and transient control, Biosystems 7:130-136 (1975).

8 J.-H. S. Hofmeyr, H. Kacser and K. J. van der Merwe, Metabolic control analysis of moiety-conserved cycles, Eur. J. Biochem. 155:631-641 (1986).

9 D. H. Irvine and M. A. Savageau, Network regulation of the immune response: alternative control points for suppressor modulation of effector lymphocytes, J. Immunol. 134:2100-2116 (1985).

10 D. H. Irvine and M. A. Savageau, Network regulation of the immune response: modulation of suppressor lymphocytes by alternative signals including contrasuppression, J. Immunol. 134:2117-2130 (1985). 
11 D. H. Irvine and M. A. Savageau, Efficient solution of nonlinear ordinary differential equations expressed in S-system canonical form, SIAM Journal on Numerical Analysis (in press).

12 H. Kacser, The control of enzyme systems in vivo: elasticity analysis of the steady state, Biochem. Soc. Trans. 11:35-40 (1983).

13 H. Kacser and J. A. Burns, The control of flux, Symp. Sac. Exp. Biol. 27:65-104 (1973).

14 H. Kacser and J. A. Burns, Molecular democracy: who shares the controls?, Trans. Biochem. Soc. 7:1149-1160 (1979).

15 H. Kacser and J. W. Porteous, Control of metabolism: what do we have to measure?, Trends Biochem. Sci. 12:5-14 (1987).

16 A. M. Ostrowski, Solution of Equations and Systems of Equations, Academic, New York, 1966.

17 W. H. Press, B. P. Flannery, S. A. Teukolsky, and W. T. Vetterling, Numerical Recipes, Cambridge University Press, Cambridge, England, 1986.

18 H. M. Sauro, J. R. Small, and D. A. Fell, Metabolic control and its analysis. Extensions to the theory and matrix method, Eur. J. Biochem. 165:215-221 (1987).

19 M. A. Savageau, Biochemical systems analysis. I. Some mathematical properties of the rate law for the component enzymatic reactions, J. Theoret. Biol. 25:365-369 (1969).

20 M. A. Savageau, Biochemical systems analysis, II. The steady state solutions for an n-pool system using a power-law approximation, J. Theoret. Biol. 25:370-379 (1969). M. A. Savageau, Biochemical systems analysis. III. Dynamic solutions using a power-law approximation, J. Theoret. Biol. 26:215-226 (1970).

M. A. Savageau, Parameter sensitivity as a criterion for evaluating and comparing the performance of biochemical systems, Nature 229:542-544 (1971).

M. A. Savageau, Concepts relating the behavior of biochemical systems to their underlying molecular properties, Arch. Biochem. Biophys. 145:612-621 (1971).

M. A. Savageau, The behavior of intact biochemical control systems, Current Topics Cell. Reg. 6:63-130 (1972).

M. A. Savageau, Optimal design of feedback control by inhibition: steady-state considerations, J. Mol. Evolution 4:139-156 (1974).

M. A. Savageau, Optimal design of feedback control by inhibition: dynamic considerations, J. Mol. Evolution 5:199-222 (1975).

27 M. A. Savageau, Biochemical Systems Analysis: A Study of Function and Design in Molecular Biology, Addison-Wesley, Reading, Mass., 1976.

28 M. A. Savageau, Growth of complex systems can be related to the properties of their underlying determinants, Proc. Nat. Acad. Sci. USA 76:5413-5417 (1979).

29 M. A. Savageau, Mathematics of organizationally complex systems, Biomed. Biochim. Acta 44:839-844 (1985).

30 M. A. Savageau, A theory of alternative designs for biochemical control systems, Biochim. Biomed. Acta 44:875-880 (1985).

31 M. A. Savageau, Control of metabolism: where is the theory?, Trends Biochem. Sci. 12:219-220 (1987).

32 M. A. Savageau and G. Jacknow, Feedforward inhibition in biosynthetic pathways: inhibition of the aminoacyl-tRNA synthetase by intermediates of the pathway, $J$. Theoret. Biol. 77:405-425 (1979).

33 M. A. Savageau and A. Sorribas, Constraints among molecular and systemic properties: Implications for physiological genetics, submitted. 
34 M. A. Savageau and E. O. Voit, Power-law approach to modeling biological systems I. Theory, J. Ferment. Technol. 60:221-228 (1982).

35 M. A. Savageau and E. O. Voit, Recasting nonlinear differential equations as S-systems: a canonical nonlinear form, Math. Biosci. 87:83-115 (1987).

36 M. A. Savageau, E. O. Voit, and D. H. Irvine, Biochemical systems theory and metabolic control theory. 1. Fundamental similarities and differences, Math. Biosci. 86:127-145 (1987).

37 M. A. Savageau, E. O. Voit, and D. H. Irvine, Biochemical systems theory and metabolic control theory. 2. The role of summation and connectivity relationships, Math. Biosci. 86:147-169 (1987).

38 A. Sorribas and R. Bartrons, Theoretic analysis of the flux control properties of a substrate cycle, Eur. J. Biochem. 158:107-115 (1986).

39 A. Sorribas and M. A. Savageau, A comparison of variant theories of intact biochemical systems. I: Enzyme-enzyme interactions and biochemical systems theory, Math. Biosci., 94:161-193 (1989).

40 A. Sorribas and M. A. Savageau, Strategies for representing metabolic pathways within biochemical systems theory: reversible pathways, Math. Biosci., 94:239-269 (1989).

41 M. E. Van Valkenburg, Network Analysis, Prentice-Hall, Englewood Cliffs, N.J., 1958.

42 E. O. Voit, Control in perspective, Trends Biochem. Sci. 12:221 (1987).

43 E. O. Voit, S-system modelling of endemic infections, Math. Modelling (in press).

44 E. O. Voit and M. A. Savageau, Power-law approach to modeling biological systems. III. Methods of analysis, J. Ferment. Technol. 60:233-241 (1982).

45 E. O. Voit and M. A. Savageau, Equivalence between S-systems and Volterra-systems, Math. Biosci. 78:47-55 (1986).

46 E. O. Voit and M. A. Savageau, Accuracy of alternative representations for integrated biochemical systems, Biochemistry 26:6869-6880 (1987).

47 H. V. Westerhoff and Y.-D. Chen, How do enzyme activities control metabolic concentrations?, Eur. J. Biochem. 142:425-430 (1984).

48 H. V. Westerhoff and D. B. Kell, Matrix method for determining steps most rate limiting to metabolic fluxes in biotechnological processes, Biotechnol. Bioeng. 30:101-107 (1987).

49 F. van Zeggeren and S. H. Storey, The Computation of Chemical Equilibria, Cambridge Univ. Press, New York, 1970. 